\title{
Contribution à l'automatisation des analyses par éléments-finis multidimensionnelles
}

\author{
Jean-Christophe Cuillière ${ }^{a}$, Sylvain Bournival et Vincent François \\ ERICCA, Département de génie mécanique, Université du Québec à Trois-Rivières, 3351 boulevard des Forges, \\ Trois-Rivières, QC, G9A 5H7, Trois-Rivières, Canada
}

Reçu le 16 décembre 2010, accepté le 29 août 2011

\begin{abstract}
Résumé - La réduction du nombre de degrés de liberté d'une étude par éléments-finis peut être obtenue en utilisant l'analyse par éléments-finis multidimensionnelle, c'est-à-dire le mélange d'éléments-finis de poutre, de coque et de volume. Cette approche multidimensionnelle permet de réduire considérablement le temps de maillage et de résolution du système. Malheureusement, la connexion d'éléments de différentes dimensions entraîne certains problèmes au niveau de la modélisation géométrique ainsi qu'au niveau de l'incompatibilité des degrés de liberté entre éléments et de la continuité entre différentes parties de maillage réalisées séparément. Cet article présente une solution complètement automatisée à ces problèmes, utilisant uniquement des éléments-finis classiques et sans recourir à l'ajout d'équations de contraintes aux interfaces entre éléments de dimensions différentes.
\end{abstract}

Mots clés : Analyse multidimensionnelle / éléments-finis / maillage / éléments de coque / éléments de poutre

\begin{abstract}
Towards the automation of mixed-dimensional finite-element analysis. When conducting a finite element analysis one way to reduce the total number of degrees of freedom is to use elements of reduced dimension such as beam and shell elements. Because of geometric complexities some models must be meshed using volume elements. If however some parts of these models fit the description of shells then a mixed-dimensional model containing shell and volume elements side by side can be used. This approach can significantly reduce the time needed to mesh and solve the system. Unfortunately, problems arise when trying to connect elements of different dimensions due to modeling problems, to incompatible degrees of freedom and to independently created meshes. This paper presents a solution to these problems based on the generation of a compatible mesh composed solely of basic elements and without the requirement of constraint equations.
\end{abstract}

Key words: Multi-dimensional analysis / finite elements / mesh generation / shell elements / beam elements

\section{Introduction}

En conception assistée par ordinateur (CAO), lors d'une analyse par éléments-finis (AEF), l'ingénieur est souvent confronté à des temps de calcul très importants et à des limites logicielles ou matérielles concernant le nombre de degrés de liberté (DDL) maximal d'une étude. La réduction dimensionnelle est une des techniques utilisées afin de minimiser le temps de calcul et/ou le nombre de DDL d'une étude donnée. En effet, l'utilisation judicieuse d'éléments de coque et de poutre, en mécanique,

a Auteur pour correspondance : cuillier@uqtr.ca permet de diminuer considérablement le nombre de DDL sans pour autant affecter la précision des résultats d'analyse.

Cependant, la réduction dimensionnelle ne s'applique pas facilement à tous les modèles $\mathrm{CAO} / \mathrm{EF}$. En effet, les parties d'un modèle les plus complexes géométriquement doivent souvent être simulées en utilisant des éléments volumiques (tétraèdres, hexaèdres, ...). Certains modèles de pièces et de structures mécaniques présentent des parties de leur géométrie qui peuvent faire l'objet d'une réduction dimensionnelle alors que d'autres parties doivent être absolument traitées en utilisant des éléments-finis volumiques. Dans d'autres cas, l'objectif 
même du calcul requiert la modélisation tridimensionnelle de certaines parties de pièces ou de structures. Quelques exemples correspondant à ce type de situation sont illustrés dans $[1,2]$. Dans ce type d'études, il est très pertinent d'utiliser l'analyse multidimensionnelle. Ceci consiste à utiliser, dans un même modèle, plusieurs types d'éléments de dimensions différentes (par exemple la combinaison d'éléments de poutre (1D), de coque (2D) et d'éléments volumiques (3D). En effet, moyennant certaines précautions sur lesquelles nous reviendrons plus loin, une étude à la fois précise et relativement rapide peut être construite sur la base du mélange de plusieurs types d'éléments.

En effet, ces analyses multidimensionnelles combinent les avantages de plusieurs types d'éléments, permettent de réduire considérablement le nombre de DDL (et donc le temps de calcul) pour les portions utilisant des éléments $1 \mathrm{D}$ et $2 \mathrm{D}$ et permettent de mailler les portions complexes géométriquement avec des éléments volumiques. Un autre avantage de l'analyse multidimensionnelle est la facilité de modification du modèle. Par exemple, dans un processus d'optimisation, un changement d'épaisseur de tôle se réduit à un simple changement de paramètre dans une analyse multidimensionnelle, alors que pour un modèle $3 \mathrm{D}$, le modèle au complet doit être modifié et remaillé.

La détermination des parties d'un composant ou d'un assemblage qui peuvent être réduites au niveau dimensionnel est encore actuellement de la responsabilité essentielle de l'ingénieur. Des méthodes, permettant de déterminer quelles parties d'une pièce sont susceptibles d'être réduites au niveau dimensionnel ont été développées afin de rendre cette tâche délicate, plus simple et plus automatique. La Medial Axis Transform ou MAT [3-5] ainsi que l'approche proposée par Chong [2] sont des exemples de progrès effectués dans ce domaine de recherche. Malheureusement, cette tâche habituellement baptisée idéalisation géométrique est loin d'être complètement (et efficacement) automatisée. Beaucoup de travail de recherche reste à faire sur ce sujet afin de disposer d'outils réellement utilisables dans un contexte industriel. Dans le cadre du travail présenté dans cet article, les modèles idéalisés sont considérés comme un intrant du processus et sont construits par le concepteur dans un système de $\mathrm{CAO}$ commercial.

La figure 1 illustre un exemple de modèle multidimensionnel (ou idéalisé). Une pièce est partiellement modélisée en utilisant des éléments volumiques pour les parties les plus complexes de la géométrie, des éléments de poutre pour les parties allongées et minces et des éléments de coque pour les parties qui peuvent être modélisées avec précision à l'aide d'une face associée à une épaisseur de paroi.

Malheureusement, dans l'application pratique, ce type d'analyse fait l'objet de plusieurs problèmes, principalement pour ce qui concerne les zones de connexion entre éléments de nature et de dimensions différentes (non continuité du maillage et incompatibilité des DDL). Tout au long de cet article, les zones où des éléments 1D

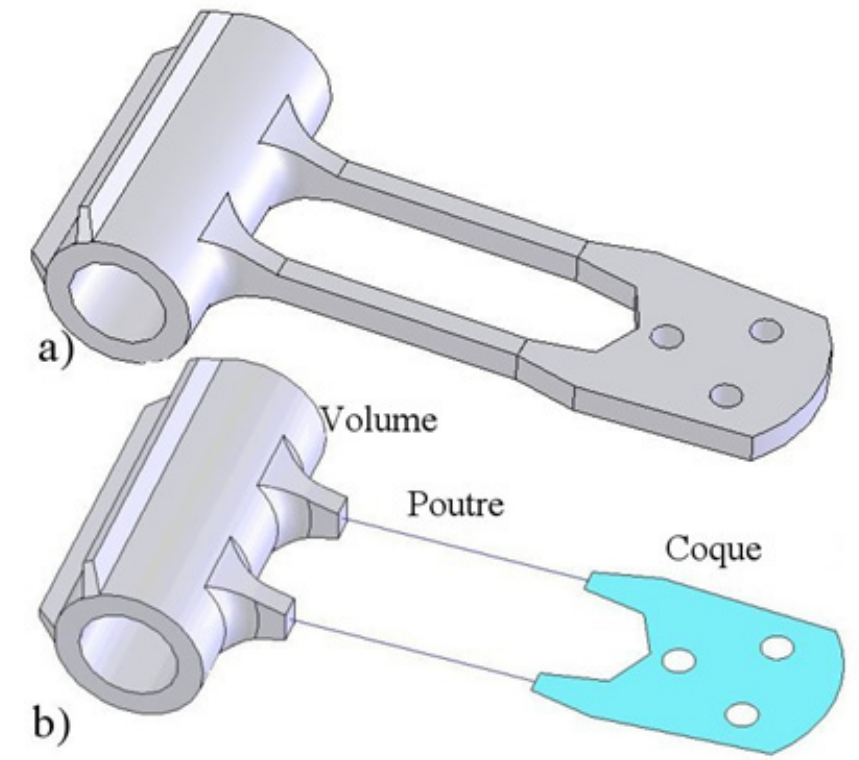

Fig. 1. Exemple de modèle multidimensionnel (a) modèle 3D, (b) modèle avec réduction dimensionnelle (modèle idéalisé).

(poutres), 2D (coques) et 3D (volumes) se connectent seront nommées interfaces dimensionnelles.

\section{Problèmes relatifs aux analyses multidimensionnelles}

La connexion d'éléments-finis de dimensions différentes au niveau d'une interface dimensionnelle fait face à quatre types de problèmes principaux. Le premier type de problème est la modélisation de la géométrie sous-jacente aux études d'éléments-finis multidimensionnelles. Le second est la continuité du maillage au niveau des interfaces dimensionnelles. Le troisième est l'éventuelle incompatibilité des degrés de liberté de part et d'autre de ces interfaces et le quatrième est la modélisation explicite de l'interface dimensionnelle.

\subsection{Modèle géométrique}

Le premier obstacle à la réalisation d'une étude multidimensionnelle est la définition d'un modèle géométrique cohérent, permettant de modéliser le mélange de plusieurs types de géométries (volumique, surfacique et filaire) et surtout de modéliser la manière dont ces différents types de géométrie sont reliés au niveau des interfaces dimensionnelles. Le modèle B-Rep ou Boundary Representation $[6,7]$ est la pierre angulaire de cette modélisation mais certaines adaptations sont néanmoins nécessaires afin de s'adapter aux spécificités des études multidimensionnelles.

Typiquement, le principe de base de la modélisation B-Rep est de définir, de manière structurée, l'enveloppe d'un objet tridimensionnel. Cette définition implique des 


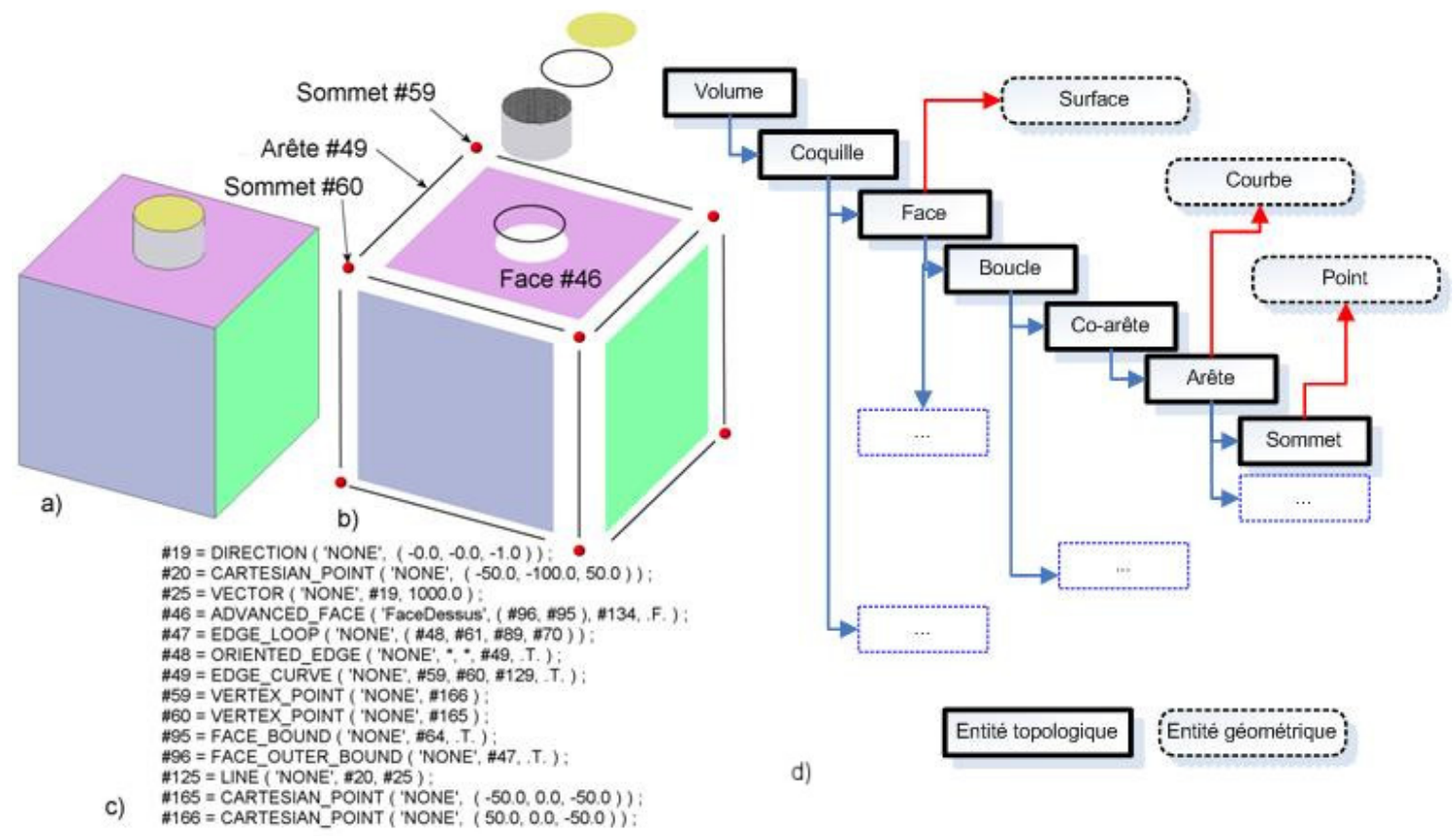

Fig. 2. Exemple d'un modèle B-Rep.

entités géométriques (points, courbes surfaces) reliées par une structure topologique basée sur la définition d'entités topologiques (sommets, arêtes, faces). La figure 2 illustre un exemple de ce type de structure sur un simple cube avec un bossage cylindrique. Le modèle part d'un pointeur sur l'entité topologique du volume qui pointe lui-même sur la ou les coquilles du volume (enveloppes externes). Chaque coquille pointe ensuite un ensemble de faces dont on définit les surfaces sous-jacentes et chaque face pointe sur une boucle d'arêtes dont on définit les courbes sousjacentes. Cette chaîne se poursuit jusqu'à la définition des sommets et des points sous-jacents qui définissent leur position (Fig. 2d). Une structure B-Rep peut être sauvée sous la forme d'un fichier (Fig. 2c) et différents formats standards peuvent être utilisés à cette fin.

À l'origine, la structure B-Rep est conçue pour décrire des pièces volumiques et elle n'est donc pas directement adaptée aux modèles comportant des composantes de dimensions différentes. Par conséquent, la structure B-Rep classique doit être adaptée dans le contexte des études multidimensionnelles.

\subsection{Continuité du maillage}

Dans notre travail, nous partons d'un modèle géométrique multidimensionnel (ou idéalisé), construit dans un système de CAO commercial comme un ensemble de composants de dimensions différentes. Sans une structure de connexion explicite, une fois maillés, ces composants sont considérés comme disjoints car le mailleur les traite indépendamment les uns des autres. Bien entendu, on obtient ainsi, en général, un maillage qui est incompatible aux interfaces dimensionnelles puisque même si les différents composants sont en contact les uns avec les autres, si les nœuds de part et d'autre d'une interface ne sont pas coïncidents, ces composants ne sont alors pas, pratiquement, reliés dans le modèle $\mathrm{EF}$. On rencontre également des problèmes aux interfaces lorsque certains nœuds sont coïncidents et d'autres non. Nous décrirons plus loin, comment ces problèmes de connexion discontinue (entre éléments de dimensions identiques et/ou différentes) peuvent être surmontés, en utilisant l'approche que nous proposons.

\subsection{Degrés de liberté incompatibles}

Le troisième problème principal, relatif aux analyses multidimensionnelles, est l'incompatibilité des degrés de liberté entre éléments de part et d'autre d'une interface dimensionnelle. En effet, les éléments-finis 3D classiques (tétraèdres, hexaèdres) présentent trois degrés de liberté par nœud (3 translations) alors que les éléments-finis classiques de type poutre et coque présentent typiquement cinq ou six degrés de liberté par nœud (trois translations et deux ou trois rotations).

Par conséquent, lorsqu'on connecte des éléments volumiques avec des éléments de coque et/ou de poutre, on rencontre, selon les circonstances, des problèmes de réalisme de liaison et ceci, même si les nœuds des composants en contact sont tous fusionnés. La figure 3 illustre un contact, sous forme de segment de droite, entre une coque et un volume. L'incompatibilité des degrés de liberté à l'interface dimensionnelle fait que la liaison à chaque nœud se comporte comme une rotule (et non comme une liaison rigide) et donc que la liaison entre la coque et le volume se comporte comme une liaison pivot (charnière) et non comme une connexion rigide. Nous décrirons plus loin, comment ces problèmes de connexion 


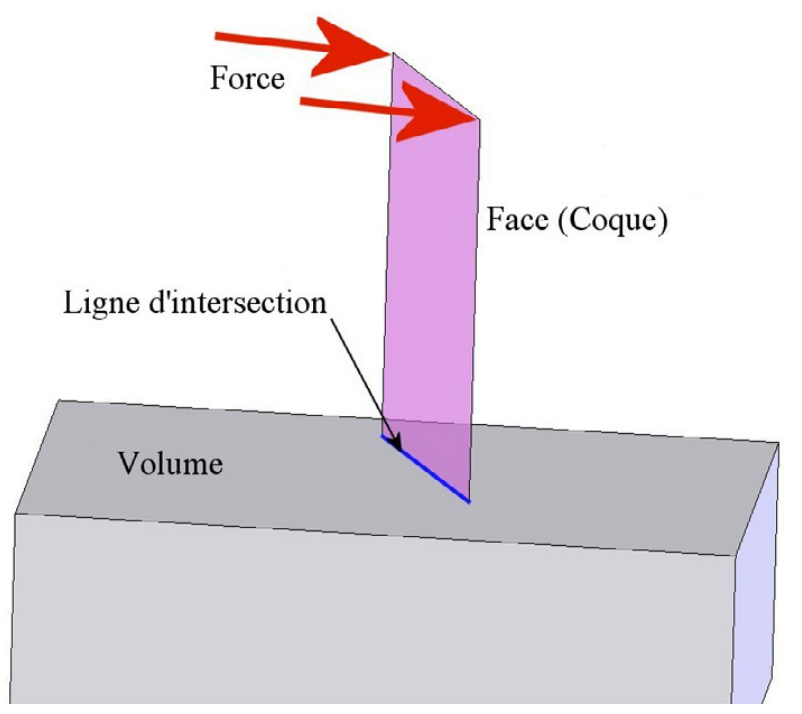

Fig. 3. Exemple d'intersection coque-volume.

inconsistante entre éléments présentant des degrés de liberté incohérents peuvent être surmontés en utilisant l'approche que nous proposons.

\subsection{Modélisation explicite de l'interface dimensionnelle}

Une fois réglés tous les problèmes mentionnés précédemment, il reste à régler le problème de la modélisation fine de la transmission des efforts (forces et moments) à travers les interfaces dimensionnelles et ceci pour tous les types de connexion.

En effet, si on reprend l'exemple de la figure 3, selon la manière dont on modélise la connexion volume-coque, la transmission des efforts peut se faire :

- sur une ligne ce qui donne soit des résultats de contrainte très irréalistes au niveau de l'interface dimensionnelle (lorsqu'il y a compatibilité des DDL) soit une incohérence due à l'incompatibilité des DDL ;

- sur une face de forme plus ou moins aléatoire, qui dépend du processus de connexion et non de la forme réelle de l'interface dimensionnelle, ce qui entraîne également des résultats de contrainte irréalistes au niveau de l'interface dimensionnelle;

- sur une face de forme dépendante de la forme détaillée de l'interface dimensionnelle, ce qui est susceptible de pouvoir en capter précisément le comportement.

En fait, de manière plus générale, pour que le champ de contrainte soit le plus précis possible au niveau de l'interface dimensionnelle et dans son voisinage, il est nécessaire de modéliser la connexion de telle manière que l'on représente à la fois fidèlement la forme de l'interface dimensionnelle mais également sa rigidité. Nous verrons dans les paragraphes suivants que la méthode que nous proposons ici permet de modéliser précisément, et de manière explicite, la forme de l'interface dimensionnelle et que cela conduit, dans ce voisinage, à des résultats en contraintes beaucoup plus précis qu'en utilisant les approches conventionnelles. Nous verrons notamment que cela est possible grâce à une adaptation spécifique de concepts de modélisation B-Rep, tels que décrits précédemment.

\section{Solutions existantes}

Quelques techniques ont été proposées dans la littérature afin de surmonter les problèmes soulevés aux paragraphes précédents. Par exemple, McCune [8] et Shim [9] proposent des approches de couplage entre éléments-finis présentant des incompatibilités de DDL utilisant un ensemble d'équations de couplage (équations de contraintes entre les degrés de liberté à l'interface) ajoutées au système, au niveau du solveur EF. Ces équations, basées sur l'équilibrage du travail mécanique de part et d'autre de l'interface dimensionnelle, permettent de surmonter les problèmes d'incompatibilité des DDL.

La méthode Arlequin [10] est également une technique pouvant être utilisée pour coupler des éléments de dimensions différentes par ajout d'équations de couplage. Cette technique permet notamment de combiner deux maillages se chevauchant en utilisant une formulation qui tient compte des deux maillages à la fois. Cette méthode peut être utilisée pour coupler des maillages de dimensions différentes qui s'intersectent.

Également, certains systèmes commerciaux de calcul par EF présentent des fonctions de couplage entre éléments présentant des incompatibilités de DDL (liaisons poutre-volume ou coque-volume). Ces fonctions sont, en fait, basées sur l'ajout d'équations de contraintes similaires à celles présentées dans le paragraphe précédent. Cependant, même si l'utilisation de ce type d'approche permet de surmonter les problèmes d'incompatibilité des DDL, la précision des résultats obtenus au niveau de l'analyse par EF est mitigée, tel que l'ont remarqué certains auteurs [8].

D'autres solutions ont été proposées par Craveur [11]. Dans le but d'éliminer l'incompatibilité des DDL, il est proposé d'ajouter des éléments poutre pour établir un lien rigide dans le cas des interfaces poutre-volume ou d'introduire des éléments-finis de connexion spécifiques. Ces propositions sont très peu documentées dans [11] et aucune piste n'est fournie quant au choix des paramètres et quant à une éventuelle automatisation du processus. Dans le cadre de la méthode proposée dans le présent article, les poutres ajoutées au niveau d'une interface dimensionnelle sont appelées mini-poutres.

L'incompatibilité au niveau des DDL peut également être abordée en utilisant des éléments volumiques présentant des DDL additionnels (deux à trois DDL en rotation afin d'être cohérent avec les éléments de poutre et de coque) en se basant, au départ, sur le principe du triangle de Allman [12]. Cet élément-fini triangulaire, conçu pour des analyses $2 \mathrm{D}$, est en fait un élément quadratique à trois nœuds, avec 3 DDL par nœud (deux translations 
Tableau 1. Trois types de connexions multidimensionnelles traitées par notre méthode.

\begin{tabular}{|c|c|c|c|c|c|}
\hline Catégorie & Arête-arête & \multicolumn{2}{c|}{ Arête-face } & \multicolumn{2}{c|}{ Face-face } \\
\hline Type & Poutre-poutre & Poutre-volume & Poutre-coque & Coque-volume & Coque-coque \\
\hline \hline Exemple & & & & &
\end{tabular}

et une pseudo-rotation). À partir de là, plusieurs types de tétraèdres présentant des DDL en rotation ont été conçus, sur la base des mêmes principes [13]. Sze, dans [14] a fait un inventaire assez exhaustif de ces types d'éléments et a mis en évidence les gains de précision potentiellement obtenus au niveau des résultats d'analyse, lorsque l'on compare ces éléments à des éléments classiques linéaires ou quadratiques. Par contre, pour ce qui est de la précision des résultats de couplage avec des éléments de poutre ou de coque, cette question n'est à peu près pas abordée dans la littérature. Nous avons évalué cette approche de couplage [15] pour de nombreuses configurations afin de la comparer avec les résultats de nos travaux. De la même manière que pour l'approche précédente (ajout d'équations de contraintes), la précision des résultats obtenus au niveau de l'analyse par EF est mauvaise, ce qui met en évidence l'intérêt pour le développement d'approches alternatives.

Nous montrerons ici comment les problèmes mentionnés ci-dessus peuvent être surmontés, en utilisant uniquement des arrangements particuliers d'éléments-finis classiques $1 \mathrm{D}, 2 \mathrm{D}$ et $3 \mathrm{D}$. Nous verrons également que l'approche proposée ici permet l'automatisation complète du processus, en partant d'un modèle idéalisé. De plus, la méthode décrite dans cet article s'adapte à tous les types de connexion, tel qu'illustré au tableau 1. Ce tableau illustre que, dans le cadre de l'approche proposée ici, tous les types de connexion impliqués dans les études multidimensionnelles se résument en fait à trois configurations possibles : les connexions arête-arête, arête-face et face-face.

\section{Méthodologie proposée}

\subsection{Modélisation géométrique}

Comme mentionné précédemment, la première problématique rencontrée est que la modélisation géométrique doit être adaptée au contexte des études multidimensionnelles. Une première étape de cette adaptation est d'étendre le concept du B-Rep par l'inclusion d'entités de coque et de poutre. La figure 4 illustre un exemple de modèle B-Rep étendu, sur un exemple multidimensionnel très simple, comportant un volume, une coque et une poutre. Ce modèle implique l'introduction de deux nouvelles entités topologiques, soit celles de coque et de poutre. Au niveau de la structure du modèle B-Rep étendu, l'entité de type coque pointe sur une coquille ouverte, qui est le pendant de la coquille d'un volume. La coquille ouverte pointe ensuite sur un ensemble de faces tout comme dans le cas de la coquille d'un volume et ces faces sont traitées comme les faces d'un volume. Quant à l'entité de type poutre, sa structure commence par un pointeur sur une boucle ouverte, qui est très semblable, au niveau structure, à la boucle d'une face de volume. Pour compléter le modèle, une propriété d'épaisseur est attachée à l'entité de type coque, sous la forme d'un attribut, alors que la définition d'une section de poutre est attachée à l'entité de type poutre, sous la forme d'un ensemble d'attributs. Pour ce qui est des poutres il est important de préciser que ces attributs vont plus loin que la simple spécification de valeurs scalaires comme dans le cas des coques (l'épaisseur de la coque). En effet, comme nous le verrons plus loin, la modélisation fine de l'interface dimensionnelle avec une poutre requiert la connaissance précise de la forme de la section de cette poutre et de son orientation. Ceci demande un accès automatisé à beaucoup plus d'information que les simples paramètres scalaires de définition des propriétés de section (aire, périmètre, matrice d'inertie de section, etc.). En particulier, ceci requiert la définition d'une bibliothèque de modèles $2 \mathrm{D}$ représentant précisément les sections de poutres (normalisées ou non).

À ce stade de la modélisation géométrique, les entités de type volume, coque et poutre restent des entités séparées. Nous verrons plus loin comment cette modélisation géométrique est adaptée, dans une deuxième 


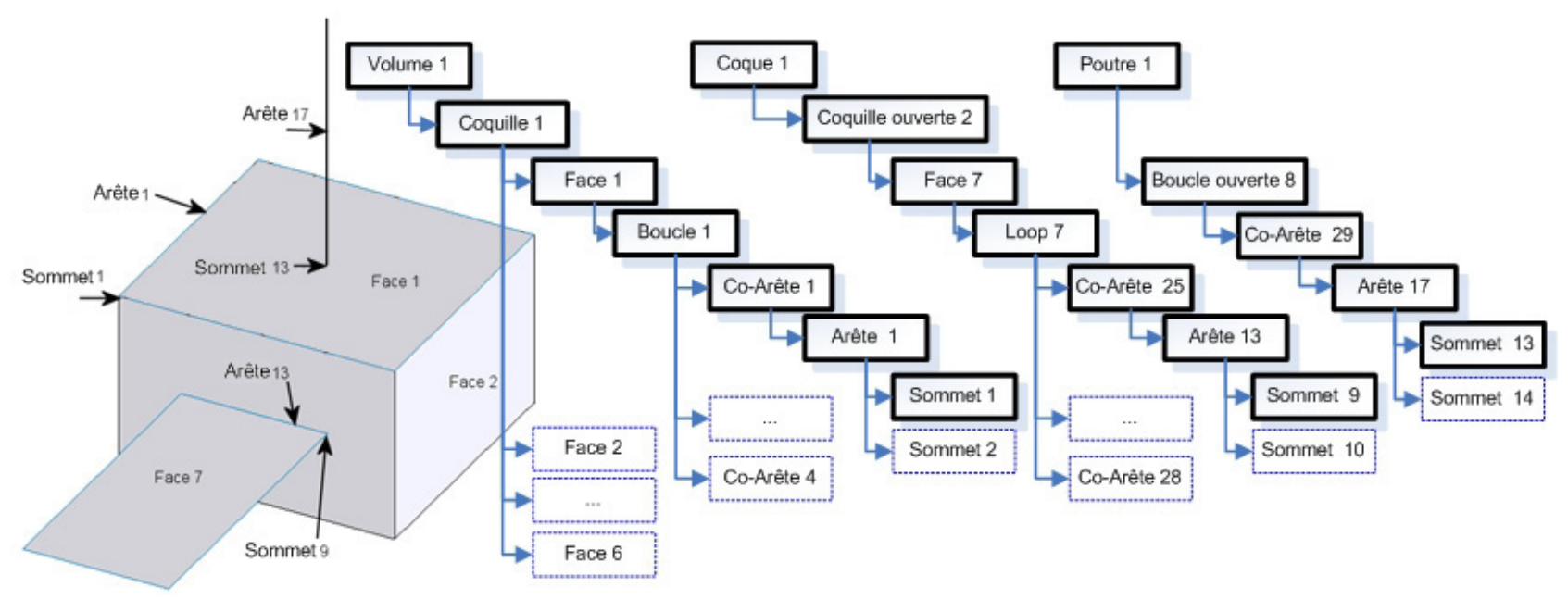

Fig. 4. Adaptation du modèle B-Rep afin d'introduire les entités de type coque et poutre.

phase, afin d'introduire des connexions entre ces éléments disjoints afin de représenter explicitement la forme précise des interfaces dimensionnelles.

\subsection{Méthodologie}

Les travaux présentés ici abordent la généralisation d'un travail préliminaire, réalisé par notre équipe, sur le problème du couplage entre éléments de poutre et éléments volumiques [15].

L'approche proposée ici n'utilise que des arrangements particuliers d'éléments-finis classiques afin de traiter toutes les catégories de connexions impliquées dans les études multidimensionnelles. Ainsi, en utilisant cette approche, les problèmes de continuité de maillage, d'incompatibilité des DDL et de représentation explicite des interfaces dimensionnelles peuvent être surmontés sans utiliser d'équations de contraintes, au niveau du solveur, ou d'éléments-finis particuliers. Cette méthodologie implique globalement trois étapes :

- le découpage de géométrie;

- l'adaptation finale du modèle géométrique;

- le maillage automatique du modèle multidimensionnel;

- l'ajout de mini-poutres.

Les deux premières étapes, sous une forme ou sous une autre, sont communes au traitement de tous les cas de connexions listés au tableau 1. L'ajout de mini-poutres est impliqué aussi bien pour les connexions arête-face que face-face du tableau 1 , avec cependant des variantes importantes selon les cas.

\section{3 Étape 1 : Découpage de géométrie}

Cette étape permet de régler, dès le début du processus, les problèmes de continuité du maillage à travers n'importe quel type d'interface dimensionnelle. Le principe utilisé étant de traiter la géométrie afin de forcer le mailleur à générer des nœuds permettant une connexion adéquate, il en résulte que l'approche est complètement indépendante du mailleur et du solveur. Pour une connexion arête-arête ce découpage est trivial et ne sera pas abordé ici puisqu'il se limite au découpage d'arêtes afin d'avoir un sommet commun. Considérons l'exemple de l'interface poutre-volume de la figure 5. Le traitement de la géométrie consiste en un découpage automatique de la zone d'interface dimensionnelle entre la poutre et le volume, basé sur la forme de la section de la poutre considérée. On commence par découper automatiquement sur la face du volume une sous-face ayant la forme de la section de la poutre, qui est ensuite ellemême découpée en deux sous-faces afin de s'assurer qu'un sommet soit présent au point de connexion avec l'élément de poutre. À partir de là, un mailleur automatique de type Delaunay ou frontal [16-18] générera automatiquement un nœud (sur la face du volume) en ce point de connexion, lors du maillage des sous-faces. D'autre part, le découpage et le maillage de ces sous-faces sont à la base de l'ajout de mini-poutres (voir plus loin). Ce processus de découpage de faces est illustré à la figure 5 . Une poutre de type $W$ ( $W$ beam), représentée par un ensemble d'éléments de poutre, se connecte à un volume en un point. Deux sous-faces sont découpées (Fig. 5c), à l'intérieur du contour de la section de type $W$, sur la face $\mathrm{du}$ volume puis maillées automatiquement (Fig. 5d). Un sommet, puis un nœud, sont générés automatiquement, selon ce processus, au point d'intersection entre la poutre idéalisée et le volume. Ainsi, le problème de continuité du maillage à l'interface dimensionnelle est résolu.

La même méthode est appliquée pour toutes les connexions arête-face. Pour ce qui est des connexions faceface (voir Tab. 1), le but du découpage de faces est de générer une arête commune à l'interface dimensionnelle entre les deux faces et de représenter explicitement l'interface dimensionnelle correspondant à l'épaisseur de la face qui se connecte sur l'autre face. Prenons l'exemple de la figure 6 où une coque se connecte sur une des faces d'un 

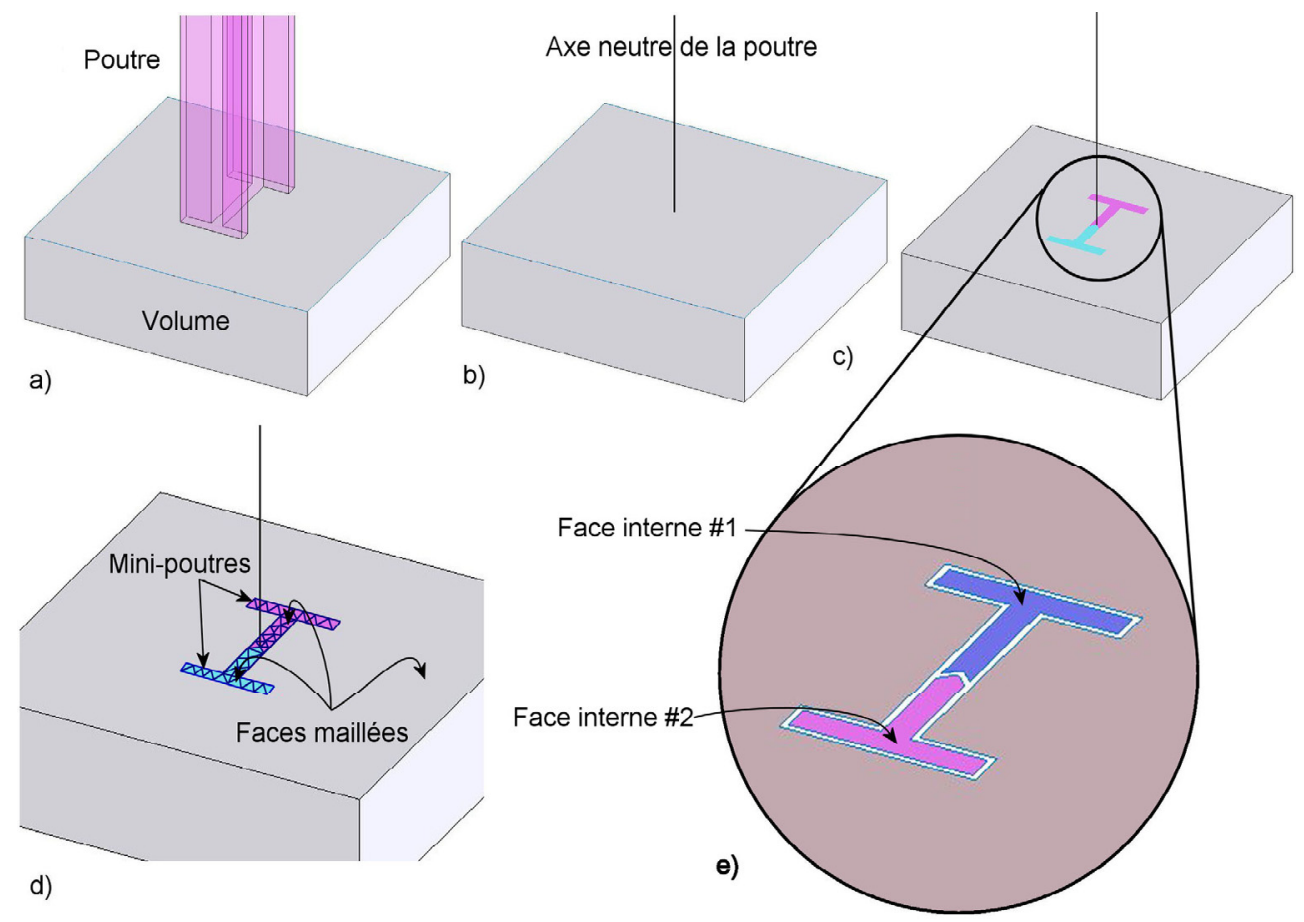

Fig. 5. (a) Modèle 3D, (b) modèle idéalisé, (c) modèle avec faces découpées, (d) ajout de mini-poutres, (e) détail de découpage de faces.

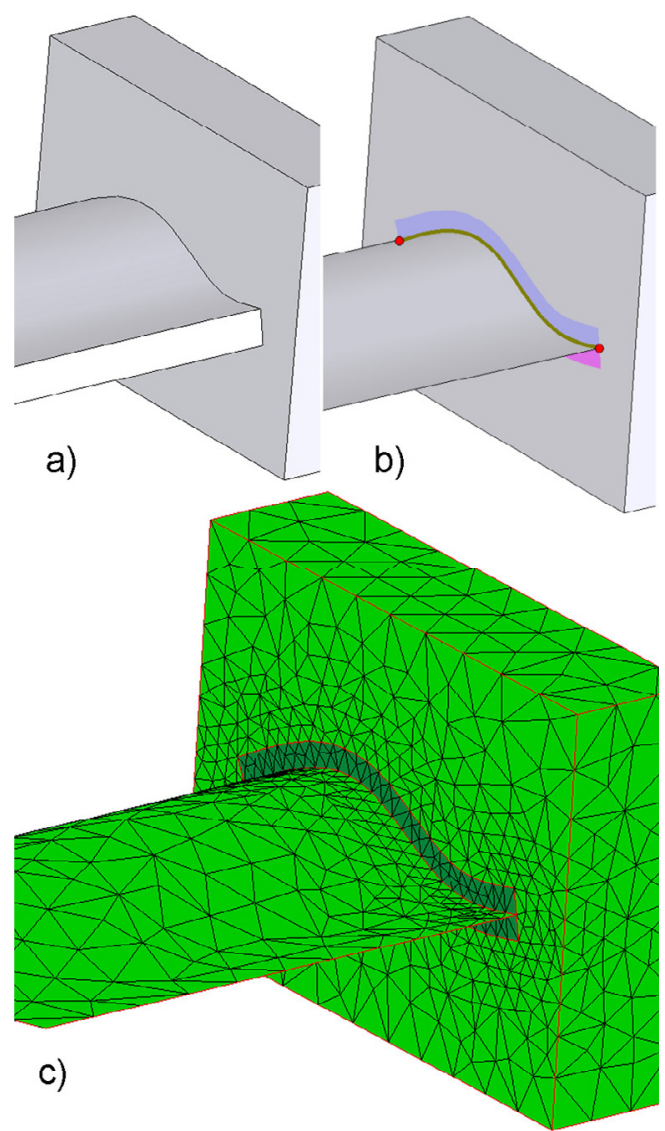

Fig. 6. Cas de connexion face-face (coque-volume), (a) modèle $3 \mathrm{D}$, (b) modèle après le découpage de faces, (c) maillage continu. volume. Le découpage de faces consiste ici à découper automatiquement la zone d'interface dimensionnelle entre la coque et le volume en fonction de l'épaisseur de paroi de la coque considérée. On commence par découper automatiquement, sur la face du volume, une sous-face ayant la forme de l'épaisseur de la coque, qui est ensuite elle-même découpée en deux sous-faces afin de s'assurer qu'une arête soit présente au lieu de connexion avec la coque. Ainsi, le découpage de face engendre par un traitement purement géométrique, comme dans le cas des connexions poutre-volume, une interface dimensionnelle cohérente avec la forme de l'intersection entre la coque et le volume (une bande ayant l'épaisseur de la coque).

Ce type de découpage est appliqué à toutes les connexions face-face. Ceci règle le problème de continuité de maillage en introduisant un modèle géométrique non manifold dans le sens où l'arête commune est commune au contour de trois faces (deux sous-faces et une face). En effet, un mailleur automatique de type Delaunay ou frontal [16-18] générera automatiquement, aux mêmes positions, des nouds communs aux trois faces. D'autre part, le découpage et le maillage des deux sous-faces sont également à la base de l'ajout de mini-poutres (voir plus loin).

\section{4 Étape 2 : Adaptation finale du modèle géométrique}

Après les opérations de découpage de faces, cette modification doit être d'abord répercutée sur la structure B-Rep adaptée du modèle géométrique ce qui permet 


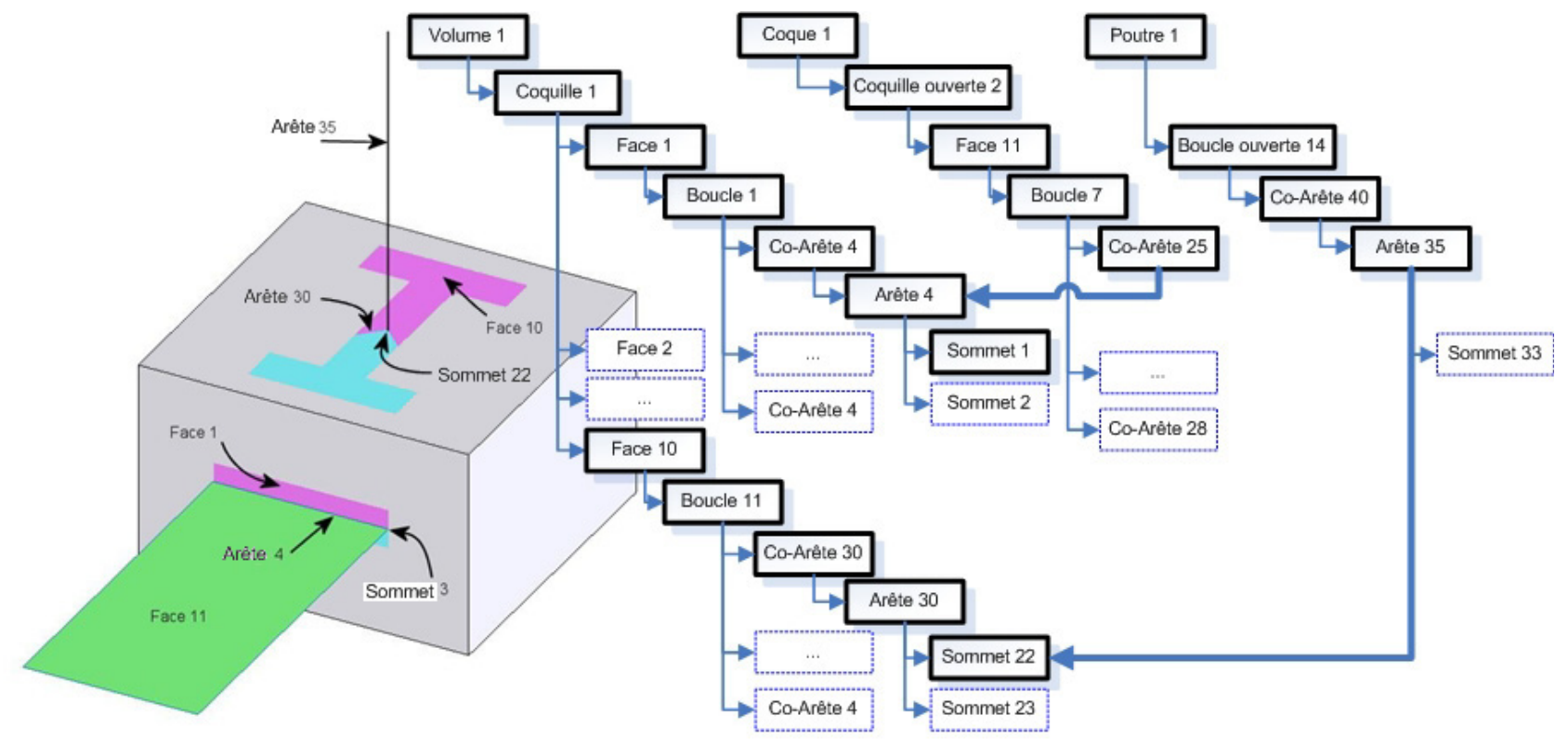

Fig. 7. Modèle B-Rep (un seul modèle).

ensuite de générer, dans cette structure, des liens entre les différentes catégories d'entités (volumes, coques et poutres).

La figure 7 reprend le même modèle que celui de la figure 4 avec, une fois le découpage de faces effectué, l'introduction, dans la structure du B-Rep adapté, des liens entre les composantes. Par exemple, une fois ces liens établis, la poutre de la figure 7 se trouve reliée au volume par le sommet commun \#22. De la même manière, la coque se trouve reliée au volume par l'arête \#4 (sousjacente aux co-arêtes \#4 du côté de la structure du volume et \#25 du côté de structure de la coque). Ainsi les structures de la coque et de la poutre se retrouvent, de manière cohérente et concise, non seulement reliées à celle du volume, mais également, la forme des interfaces dimensionnelles se retrouve modélisée explicitement dans un modèle global et continu de la géométrie multidimensionnelle.

Cette adaptation de la géométrie garantit que, par exemple, au point de connexion poutre-volume, puisqu'il n'y a qu'un sommet, il n'y aura qu'un seul nœud à cet endroit et que le maillage sera bien continu à cet endroit. De la même manière, le maillage de l'arête commune \#4 sera commun aux maillages de la coque et du volume, ce qui entraîne également que le maillage sera parfaitement continu à cet endroit. D'autre part, la représentation explicite des interfaces dimensionnelles permet de solutionner facilement et efficacement le problème de l'incompatibilité des degrés de liberté, tel que présenté dans les paragraphes suivants.

\section{5 Étape 3 : Maillage automatique du modèle multidimensionnel}

Une fois le modèle géométrique multidimensionnel traité selon les étapes précédentes, un mailleur automatique classique de type Delaunay ou frontal [1618] peut être appliqué à l'ensemble des entités du modèle. Comme expliqué précédemment et comme cela sera illustré plus loin par un ensemble d'exemples d'application, ce traitement et cette modélisation spécifique de la géométrie garantit la continuité du maillage au niveau des interfaces dimensionnelles, et ceci pour tous les cas de connexion mentionnés au tableau 1 .

Pour ce qui est de la suite du processus, les problèmes d'incompatibilité entre DDL ainsi que la modélisation de la rigidité de l'interface dimensionnelle sont abordés en utilisant l'ajout de mini-poutres. Ces mini-poutres sont des éléments-finis de poutre classiques qui sont utilisés spécifiquement à des fins de connexion au niveau des interfaces dimensionnelles arête-face et face-face (voir Tab. 1). Étant donné que l'ajout de mini-poutres n'est pas tout à fait le même dans les cas de connexions arête-face que dans le cas des connexions face-face, nous aborderons ces processus à travers deux sections séparées.

\section{Connexions arête-face}

\subsection{Ajout de mini-poutres}

Les problèmes d'incompatibilité des DDL entre les éléments de poutre (et de coque dans la section suivante) et les éléments volumiques ainsi que la modélisation de la rigidité de l'interface dimensionnelle sont abordés en ajoutant des mini-poutres sur les arêtes du maillage des interfaces dimensionnelles arête-face (et face-face dans la section suivante). Ces mini-poutres sont générées sur les arêtes du maillage des interfaces dimensionnelles ainsi que sur leurs contours (représentant par exemple le contour de la section d'une poutre). Ces mini-poutres sont ensuite assemblées avec le reste du maillage. Ainsi, cette 
triangulation de mini-poutres réalise une liaison plus ou moins rigide (selon les propriétés des mini-poutres) entre les éléments de poutre (et de coque tel qu'illustré plus loin) et la face de connexion. Cette liaison (en plus de régler le problème d'incompatibilité des DDL) a la forme de la section de la poutre (ou de la coque). Il est à noter que cette distribution de mini-poutres a été étudiée soigneusement et est le résultat de nombreux essais de validation du concept [19] tel que présenté ci-dessous pour des connexions poutre-volume.

\subsection{Optimisation}

Tel que mentionné ci-dessus, la distribution des minipoutres a fait l'objet d'une étude, au cours de laquelle différentes configurations de placement et de propriétés des mini-poutres ont été testées sur un exemple de connexion poutre-volume, soumis à différents cas de sollicitation mécanique. Le principe de ces tests de validation a été de comparer, pour chaque configuration, les résultats d'analyse par EF obtenus avec un modèle idéalisé et traité avec notre méthode, avec les résultats d'analyse par EF obtenus pour un modèle de référence, complètement maillé en 3D avec des tétraèdres quadratiques et avec une taille d'éléments extrêmement petite. Concernant l'influence de la rigidité des mini-poutres sur la précision des résultats obtenus, une étude plus approfondie reste à faire pour s'adapter à tous les cas de chargement. Cependant, l'utilisation de mini-poutres très rigides s'est avérée une solution très acceptable. Ceci consiste, en fait, à imposer une surface de connexion rigide, ayant la forme de la section de la poutre, au niveau de l'interface dimensionnelle.

\subsection{Comparaison et évaluation}

La présente section illustre une partie des tests de validation effectués pour évaluer les paramètres de la méthode et pour la comparer aux méthodes alternatives. L'exemple proposé ici (tiré de [20]) compare un modèle où une poutre (de longueur $254 \mathrm{~mm}$ ) à section carrée (38,1 mm de côté) vient se connecter sur un prisme rectangulaire volumique $(254 \mathrm{~mm} \times 127 \mathrm{~mm} \times 102 \mathrm{~mm})$. La charge de $4459 \mathrm{~N}$ est appliquée à l'extrémité de la poutre horizontalement. Le matériau du bloc et de la poutre (acier) a un module de Young de $210000 \mathrm{MPa}$ et un coefficient de Poisson de 0,28. Le modèle est illustré à la figure $8 \mathrm{a}$ et la contrainte est évaluée sur une centaine de points situés sur une ligne d'évaluation (identifiée sur le modèle). Les figures $8 b$ et c présentent des graphiques illustrant la variation de la contrainte de Von Mises le long de cette ligne d'évaluation. Quatre approches multidimensionnelles sont comparées au modèle de référence (modèle 3D maillé très finement avec des éléments quadratiques), soit celle décrite dans cet article, la méthode de Craveur [11], une méthode implantée dans un logiciel commercial utilisant des équations de contraintes, et une méthode utilisant des éléments tétraédriques avec degrés de libertés en rotation [13]. Pour ce qui est de la figure 9 , on utilise le même principe sur une comparaison des différentes approches dans le cas d'une connexion avec une poutre carrée creuse. On peut constater sur les graphiques des figures 8 et 9 que notre approche capte nettement mieux que les autres méthodes le comportement du modèle de référence. Ceci est particulièrement remarquable lorsque l'on raffine le maillage car notre méthode n'engendre pas de discontinuités au centre (au point de connexion) et concentre la contrainte là où elle doit l'être, soit sur les contours de la section de la poutre. Nous avons réalisé une comparaison quantitative des différents résultats obtenus aux figures 8 et 9 basée sur la norme $L_{2}$ (représentant la distance de chaque courbe de contrainte par rapport à la courbe de contrainte de référence). Le tableau 2 compare ces valeurs (en se limitant à une zone située au voisinage de l'interface dimensionnelle) pour les différentes approches dans le cas des figures 8 et 9 . On constate clairement que les valeurs obtenues en utilisant notre approche sont beaucoup plus faibles, ce qui signifie que la courbe obtenue colle beaucoup mieux à la courbe de référence.

\subsection{Raideur des mini-poutres et énergie de jonction}

Dans l'état actuel de développement de la méthode, l'utilisation de mini-poutres très rigides s'avère un très bon compromis puisque cela permet d'avoir des résultats de contrainte dans les zones des interfaces dimensionnelles assez réalistes pour tous les cas de sollicitation de ces interfaces. Une étude plus poussée pourrait néanmoins être menée afin d'améliorer encore davantage la précision de la méthode, surtout au niveau de la répartition des contraintes au niveau des interfaces dimensionnelles. Un critère potentiellement utilisable est d'analyser l'énergie de déformation associée à l'ensemble des mini-poutres. En effet, cette énergie est influencée par les propriétés de raideur des mini-poutres et doit théoriquement être négligeable vis-à-vis de l'énergie de déformation du reste de la structure étudiée puisque les mini-poutres représentent un ajout au modèle et n'ont donc pas d'existence réelle. On peut observer une corrélation directe entre la précision des résultats (en contrainte et en flèche) et la valeur de l'énergie de déformation associée à l'ensemble des mini-poutres. Par exemple, pour la structure et le cas de charge illustrés à la figure 8, le tableau 3 illustre les résultats obtenus pour différentes valeurs de moment d'inertie des mini-poutres (en fait un seul des deux moments d'inertie a une influence) et différentes valeurs de l'aire de section des mini-poutres. Le tableau fournit l'énergie de déformation totale (excluant les minipoutres) et le pourcentage de l'énergie de déformation associée aux mini-poutres comparée à celle du reste de la structure. Il fournit également la flèche horizontale à l'extrémité de la poutre ainsi qu'une comparaison quantitative des résultats de contrainte dans la zone de l'interface dimensionnelle basée sur la norme $L_{2}$ (représentant la distance de chaque courbe de contrainte par rapport à la courbe de contrainte de référence). La figure 10, 


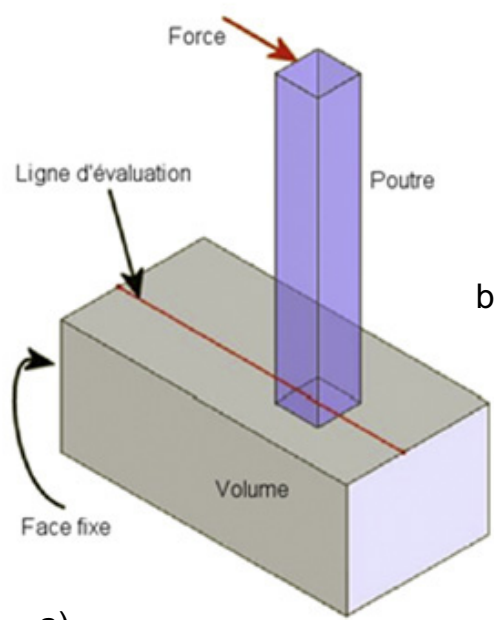

a)

c)
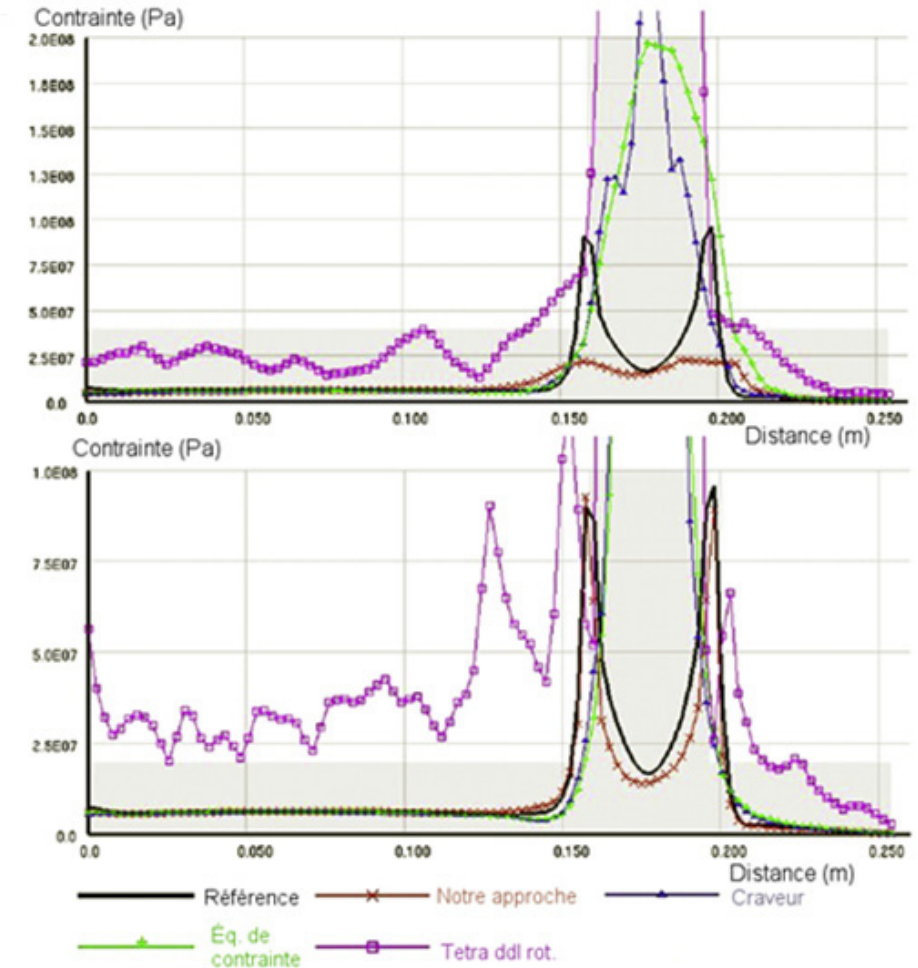

Fig. 8. Évaluation et comparaison des approches, (a) modèle de référence, (b) modèle avec un maillage grossier, (c) modèle avec un maillage raffiné.

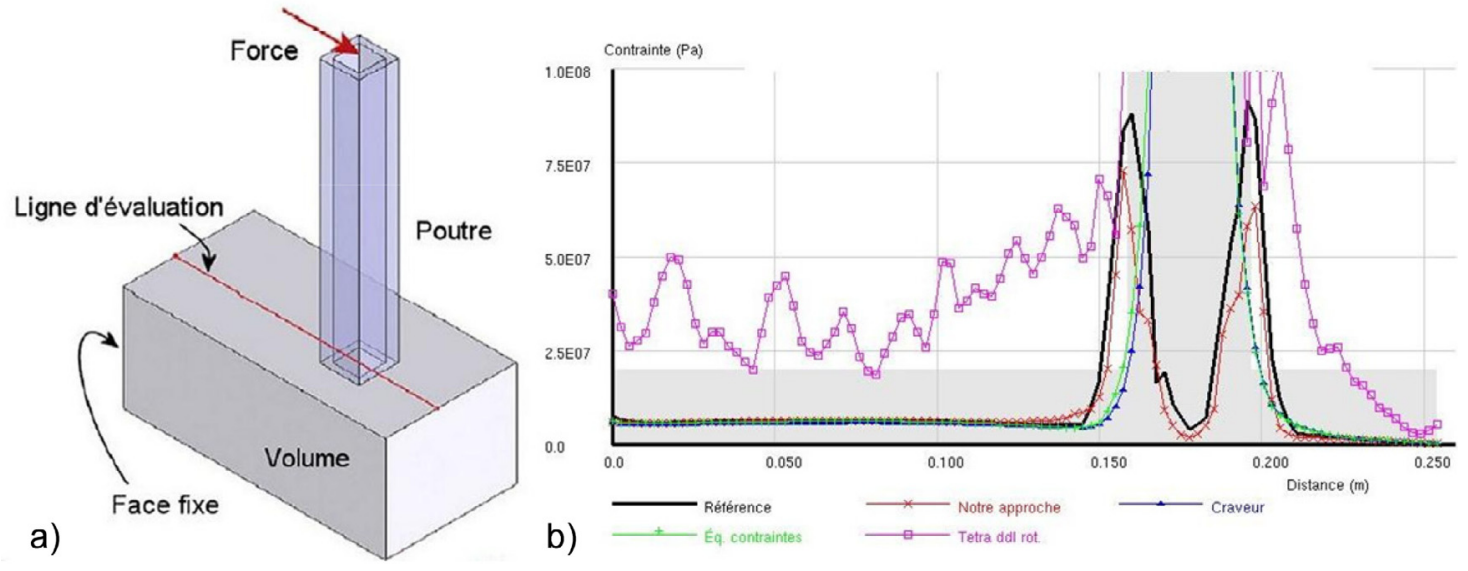

Fig. 9. Évaluation et comparaison des approches, (a) modèle de référence, (b) comparaison.

Tableau 2. Comparaison des courbes de la figure 8 avec l'utilisation de la norme $L_{2}$.

\begin{tabular}{cccc}
\hline Distance $L_{2}\left(\times 10^{7} \sqrt{m} \mathrm{~Pa}\right)$ & $\begin{array}{c}\text { Modèle grossier } \\
\text { figure } 8\end{array}$ & $\begin{array}{c}\text { Modèle raffiné } \\
\text { figure } 8\end{array}$ & $\begin{array}{c}\text { Modèle } \\
\text { figure } 9\end{array}$ \\
\hline Notre approche & 0,7 & 0,3 & 0,4 \\
Approche de Craveur [11] & 2,5 & 900,8 & 2936,2 \\
Équations de contrainte & 2,6 & 2865,8 & 3312,3 \\
Tétra. avec ddl en rotation [13] & 15,2 & 2819,5 & 6063,7 \\
\hline
\end{tabular}




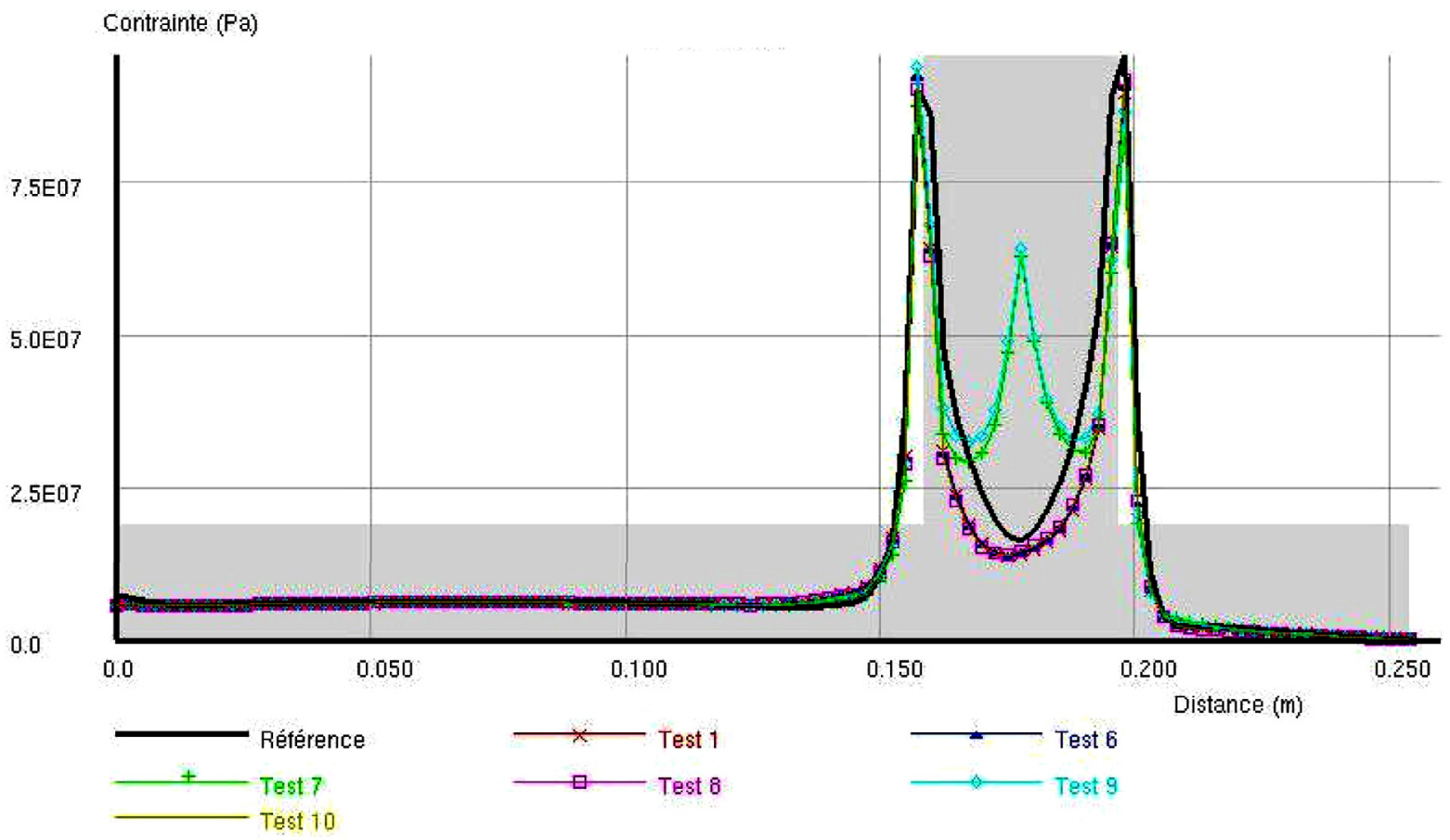

Fig. 10. Comparaison des contraintes avec la référence pour les cas du tableau 3.

Tableau 3. Comparaison des énergies, contraintes et flèches pour différentes propriétés des mini-poutres.

\begin{tabular}{ccccccc}
\hline & $\begin{array}{c}\text { Inertie } I_{z} \\
\text { section }\left(\mathrm{m}^{4}\right)\end{array}$ & $\begin{array}{c}\text { Aire } \\
\text { section }\left(\mathrm{m}^{2}\right)\end{array}$ & $\begin{array}{c}\text { Énergie } \\
\text { structure }(\mathrm{J})\end{array}$ & $\begin{array}{c}\text { \% Énergie } \\
\text { mini-poutres }\end{array}$ & $\begin{array}{c}\text { Distance } L_{2} \\
\left(\times 10^{7} \sqrt{m} \mathrm{~Pa}\right)\end{array}$ & $\begin{array}{c}\text { Flèche } \\
(\mathrm{m})\end{array}$ \\
\hline Référence & $\mathrm{NA}$ & $\mathrm{NA}$ & 1,75 & $\mathrm{NA}$ & $\mathrm{NA}$ & $7,82 \times 10^{-4}$ \\
Test 1 & $10^{-2}$ & $10^{-2}$ & 1,71 & $2,8 \times 10^{-4}$ & 0,27 & $7,69 \times 10^{-4}$ \\
Test 6 & $10^{-4}$ & $10^{-5}$ & 1,71 & $3,6 \times 10^{-2}$ & 0,27 & $7,70 \times 10^{-4}$ \\
Test 7 & $10^{-9}$ & $10^{-9}$ & 1,72 & 33 & 0,43 & $10,32 \times 10^{-4}$ \\
Test 8 & $10^{-7}$ & $10^{-7}$ & 1,71 & $3,4 \times 10^{-1}$ & 0,27 & $7,72 \times 10^{-4}$ \\
Test 9 & $10^{-9}$ & $10^{-5}$ & 1,72 & 33 & 0,42 & $10,31 \times 10^{-4}$ \\
Test 10 & $10^{-7}$ & $10^{-9}$ & 1,71 & $3,4 \times 10^{-1}$ & 0,27 & $7,72 \times 10^{-4}$ \\
\hline
\end{tabular}

quant à elle, fournit le détail de l'évolution des contraintes de Von Mises le long de la ligne d'évaluation illustrée à la figure 8 avec la comparaison avec la référence (pour chaque cas du Tab. 3). On constate que, pour ce cas de chargement, l'aire de section n'influe pas sur les résultats mais qu'il y a un seuil sur le moment d'inertie $I_{\mathrm{z}}$ au-delà duquel l'énergie de déformation des mini-poutres n'est plus négligeable, ce qui détériore les résultats, à la fois en flèche et en contrainte.

\section{Connexions face-face}

\subsection{Ajout de mini-poutres}

Ici aussi, les problèmes d'incompatibilité des DDL entre les éléments de coque et les éléments volumiques ainsi que la modélisation de la rigidité de l'interface dimensionnelle sont abordés en ajoutant des mini-poutres sur les arêtes du maillage des interfaces dimensionnelles face-face en se basant sur les arêtes de la triangulation des sous-faces. Comme dans le cas des connexions arêteface, cette méthode modélise l'interface dimensionnelle de manière plus réaliste puisque la connexion face-face se fait sur une face (de largeur égale à l'épaisseur de la coque) plutôt que sur une arête (ce qui engendrerait des concentrations de contraintes irréalistes au niveau de cette arête de connexion).

L'ajout de ces mini-poutres, tout comme dans le cas de connexions poutre-volume, est une opération relativement simple puisqu'il suffit de superposer une mini-poutre sur chaque segment de maillage appartenant à la triangulation des sous-faces de l'interface dimensionnelle. Pour ce qui concerne les propriétés mécaniques de ces minipoutres, dans le cas des connexions arête-face, il a été mentionné que l'utilisation de mini-poutres très rigides s'est avérée une solution très acceptable. Cette solution ne peut cependant pas être utilisée dans le cas des connexions face-face en raison du fait que la section de la coque, au niveau de l'interface dimensionnelle, doit pouvoir se déformer, le cas échéant, dans le plan de l'interface dimensionnelle, tel qu'illustré à la figure 11. Cette figure 


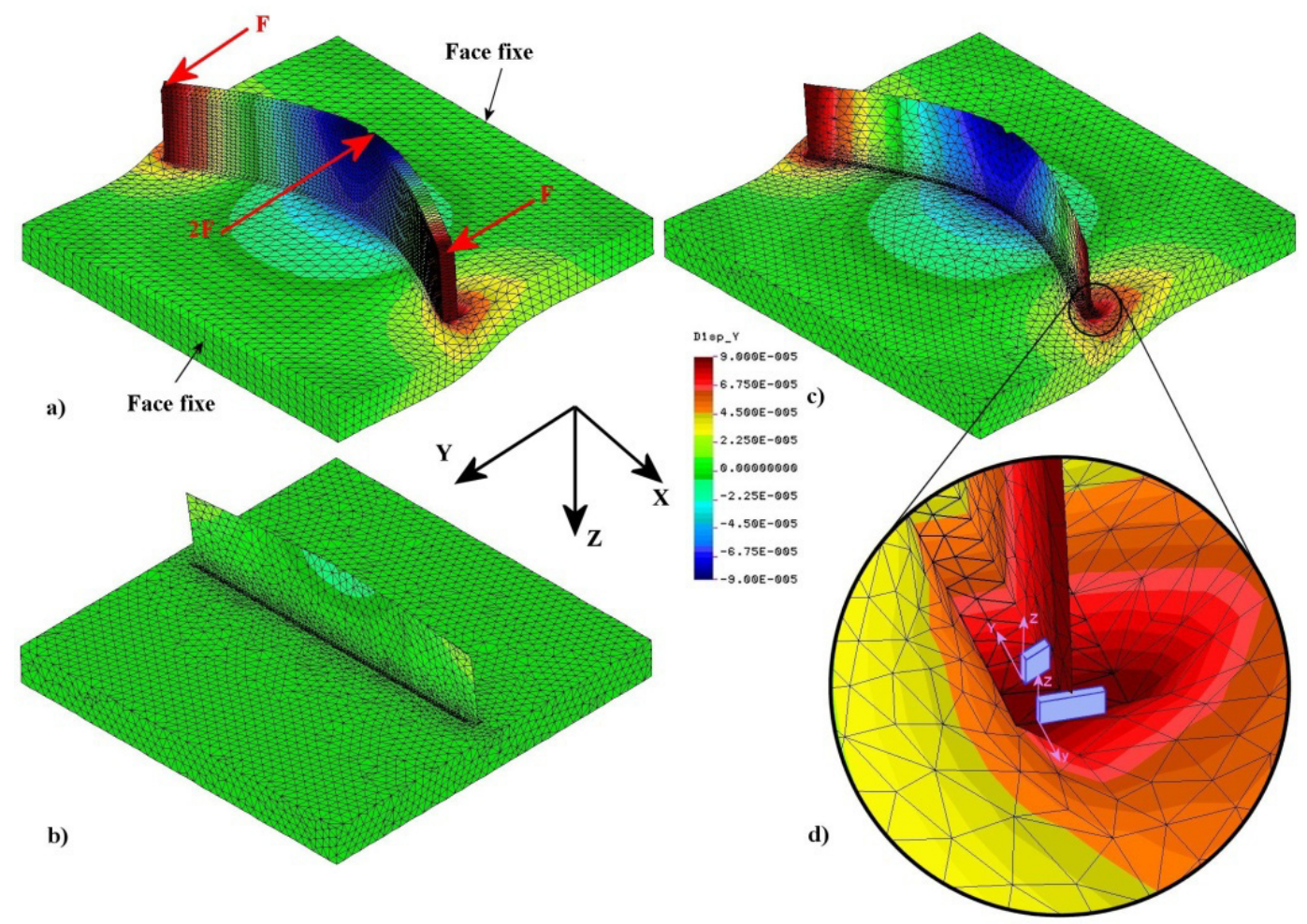

Fig. 11. Résultats (déplacements dans la direction de $Y$ ) pour une connexion coque-volume. (a) Avec un maillage 3D, (b) avec des mini-poutres infiniment rigides, (c) avec des mini-poutres orientées, (d) illustration de l'orientation de deux mini-poutres.

présente un cas de chargement d'une connexion coquevolume où les forces appliquées sur la coque engendrent la flexion de la coque et de l'interface dimensionnelle. La figure 11a présente le résultat obtenu en utilisant un maillage volumique très fin utilisé comme référence. Si on utilise des mini-poutres infiniment rigides (Fig. 11b), la coque ne peut pas se déformer adéquatement au niveau de la connexion et les résultats obtenus sont irréalistes.

Pour résoudre ce type de problèmes, nous avons introduit dans la méthode, telle qu'appliquée aux connexions arête-face, des mini-poutres orientées (Fig. 11c) à la place de mini-poutres très rigides dans toutes les directions. Le concept d'orientation de ces mini-poutres se traduit par le fait que les propriétés de section sont ajustées différemment selon les axes de section. En effet, ces minipoutres orientées présentent un moment d'inertie très grand autour d'un axe de section (l'axe $y$ sur la Fig. 11d) et pratiquement nul autour de l'autre axe de section (l'axe $z$ sur la Fig. 11d), ainsi qu'une aire de section pratiquement nulle. Par conséquent, la rigidité de la jonction dans le plan de l'interface dimensionnelle est produite directement par les rigidités combinées de la coque et du volume, ce qui est beaucoup plus réaliste.

La figure 12 présente des résultats de contrainte de Von Mises obtenus sur un modèle très simple où une partie de coque a été modélisée avec des éléments 3D alors que l'autre partie a été modélisée en utilisant des éléments de coque. On peut observer en particulier, sur cette figure, la continuité du champ de contraintes à travers l'interface dimensionnelle aussi bien en traction qu'en flexion.

\section{Exemples}

\section{1 Étude détaillée de joints d'une structure de levage}

La figure 13 illustre le modèle 3D solide du premier exemple complet sur lequel la méthode présentée dans cet article est appliquée. Il s'agit d'une structure de levage en acier d'environ 100 mètres de largeur pour 50 mètres de hauteur sur laquelle on applique différentes charges.

Le modèle idéalisé est présenté à la figure 14 . L'objectif de calcul est d'évaluer assez précisément les contraintes dans certaines jonctions entre poutres en utilisant un modèle multidimensionnel mélangeant des éléments de poutre avec des éléments volumiques. On utilise également une symétrie compte tenu de la symétrie du modèle et du chargement. La figure 15a présente tous les points de jonction calculés pour la connexion des mini-poutres alors que les figures $15 \mathrm{~b}$ et c présentent les résultats de contrainte de Von Mises obtenus en focalisant sur une zone de jonction entre quatre poutres se rencontrant à angle. Grâce à la méthode présentée ici, la géométrie complexe de la zone d'intersection entre les quatre poutres peut être facilement étudiée à l'aide d'éléments volumiques, alors que les poutres sont modélisées en utilisant des éléments de poutre. 

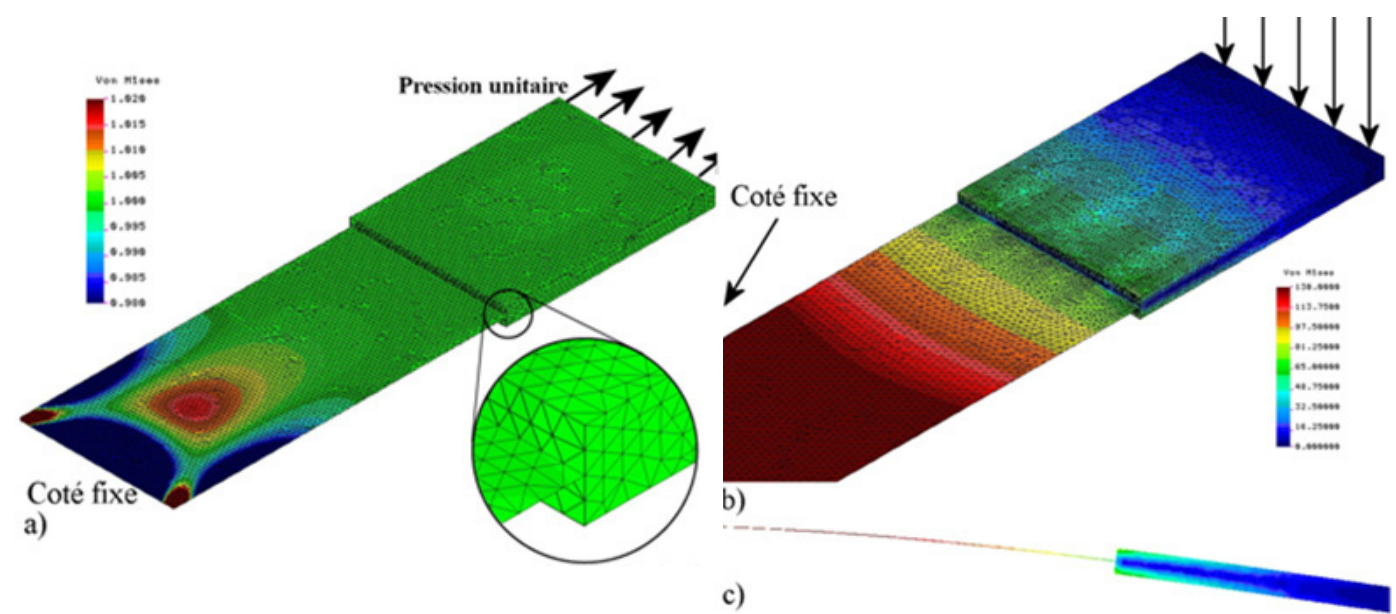

Fig. 12. Résultats de contraintes de Von Mises pour une coque partiellement maillée avec des éléments volumiques et des éléments de coque. Les contraintes sur la face supérieure sont affichées pour les éléments de coque. (a) Charge axiale, (b) charge en flexion (c).

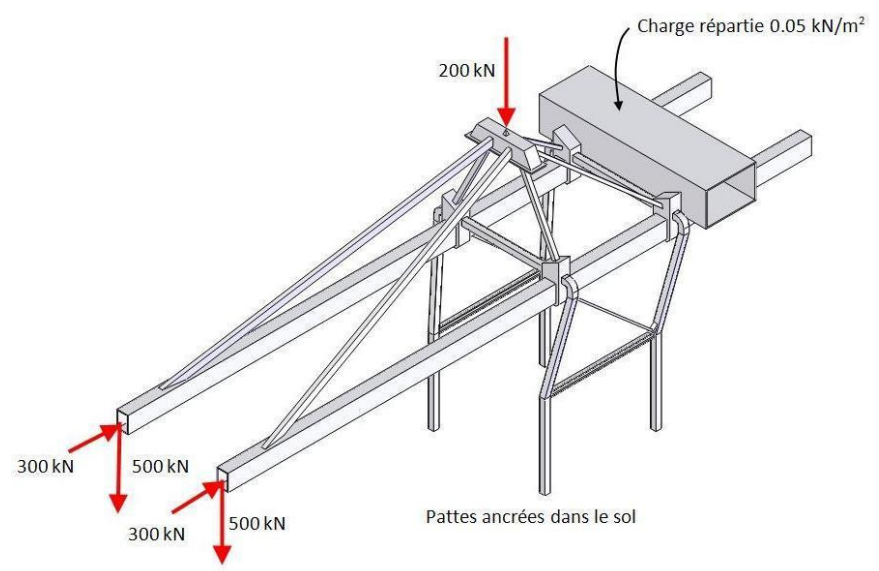

Fig. 13. Modèle solide d'une structure de levage.

Cette figure $15 \mathrm{c}$ illustre également la manière particulière dont sont traitées les poutres présentant des sections creuses. En effet, nous avons dû développer un schéma de connexion spécifique pour ce type de sections [19], pour lesquelles l'axe neutre (et donc le point de connexion théorique entre poutre et volume) n'est pas dans la matière de la poutre, ce qui est ce cas, par exemple, pour les sections tubulaires et les sections en C. Pour ces configurations particulières, une mini-poutre supplémentaire est ajoutée entre l'extrémité de la poutre (le point de connexion théorique) et un point situé sur les sous-faces représentant la section de la poutre (voir par exemple la connexion avec la section tubulaire carrée sur la Fig. 15c).

\subsection{Support}

Ce deuxième exemple démontre le potentiel de la méthode par son utilisation dans la modélisation d'un support à 6 pattes (Fig. 16). 5 pattes sont modélisées

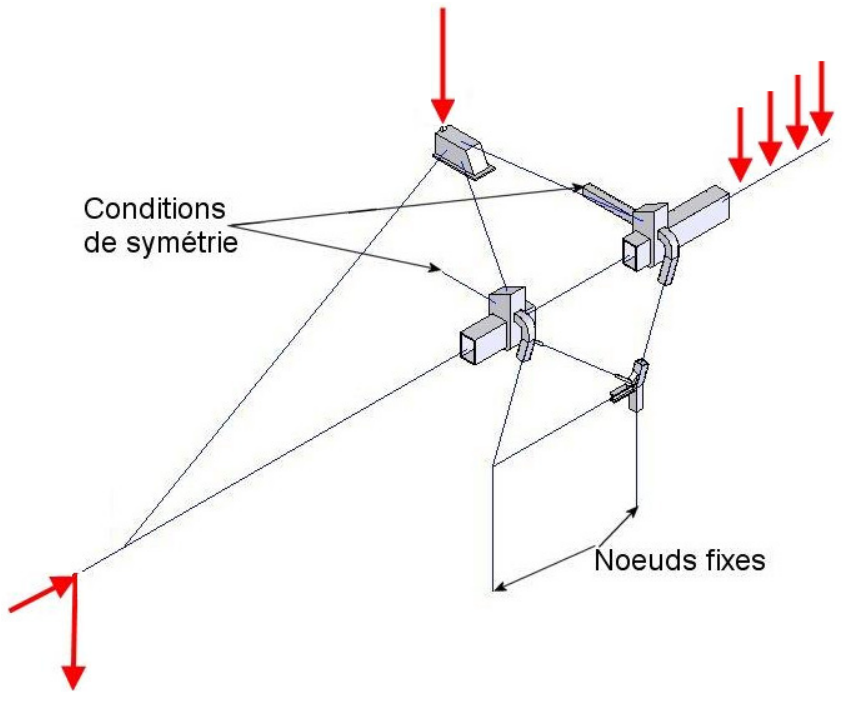

Fig. 14. Idéalisation du modèle de structure de levage.

partiellement avec des éléments volumiques et partiellement avec des éléments-finis de coque, la longueur modélisée avec des éléments volumiques étant différente pour chaque patte (Fig. 16b). La sixième patte est entièrement modélisée avec des éléments-finis de coque. Une pression unitaire est appliquée sur le bloc central alors que l'extrémité de chaque patte est encastrée. On constate que, bien que chaque patte soit maillée de façon différente, le résultat en contrainte est similaire sur chacune des pattes (Fig. 17).

Le tableau 4 résume certaines données quantitatives (nombre de nœuds et de DDL et déplacement vertical maximal) concernant le maillage et les résultats obtenus sur 3 modèles. Le premier est le modèle multidimensionnel illustré à la figure 16. Le second est un modèle entièrement maillé à l'aide d'éléments tétraédriques quadratiques et très raffiné. Le troisième et dernier modèle 


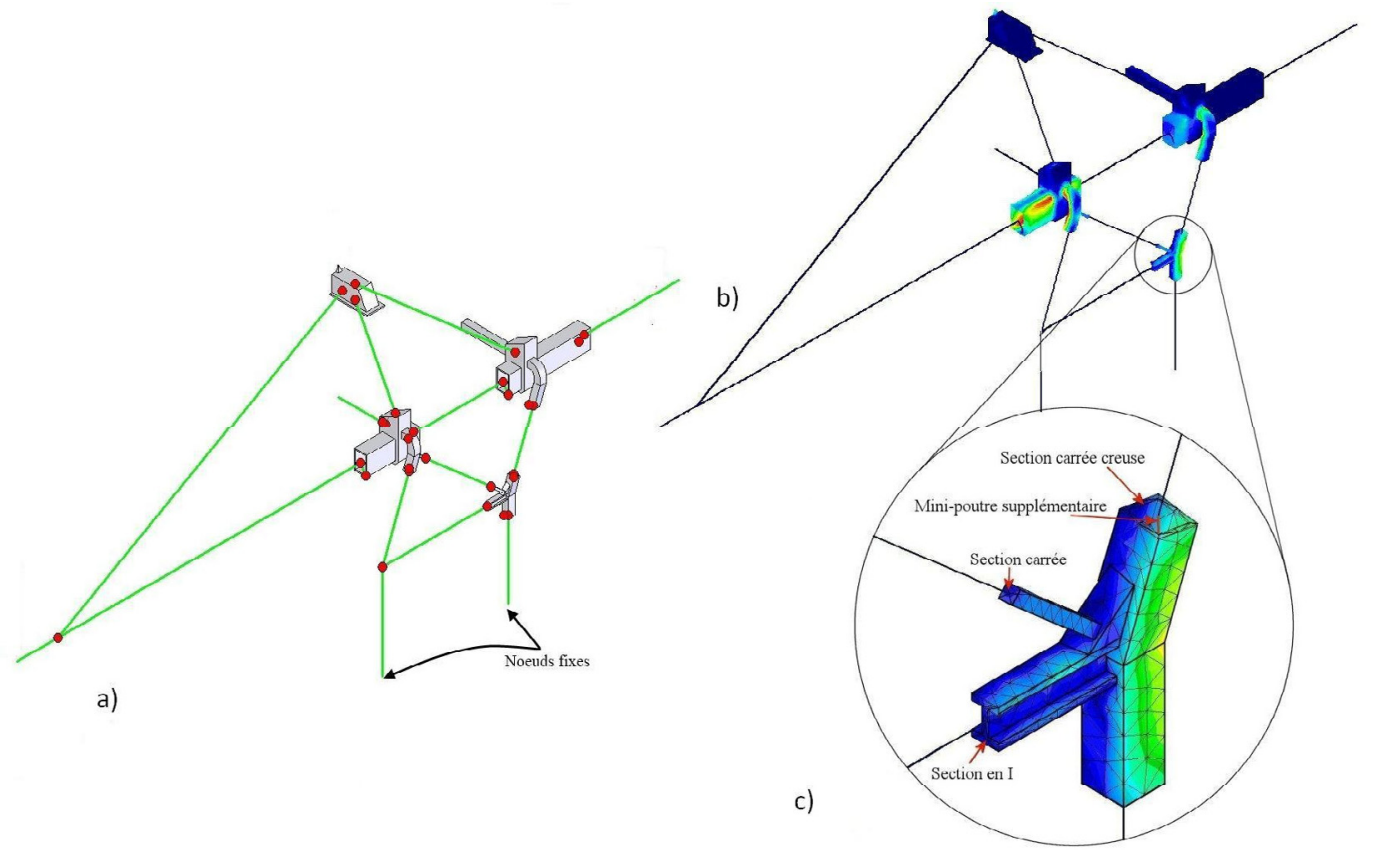

Fig. 15. (a) Points de jonction générés, (b) et (c) contraintes de Von Mises avec un zoom sur une zone d'intérêt.

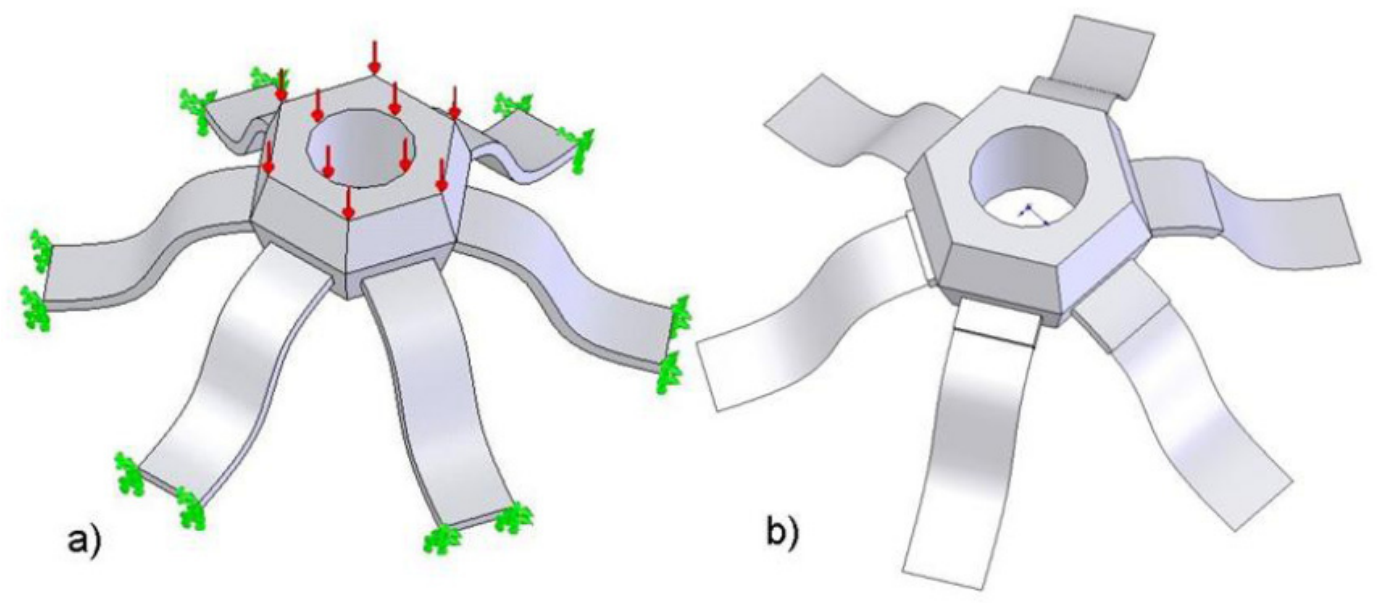

Fig. 16. (a) Modèle solide avec conditions imposées, (b) idéalisation.

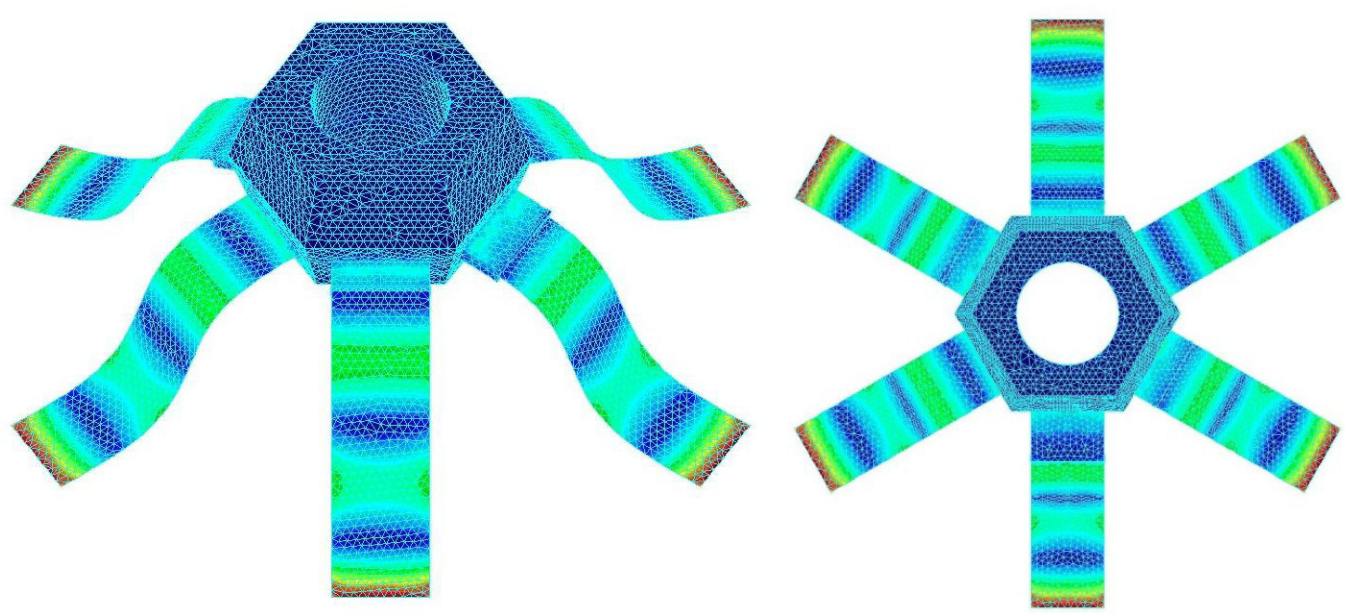

Fig. 17. Contraintes obtenues. 
Tableau 4. Comparaison de 3 différents modèles.

\begin{tabular}{cccc}
\hline & Multidimensionnel & 3D raffiné & 3D grossier \\
\hline Nb. éléments & 23171 & 88048 & 32154 \\
Nb. DDL & 20295 & 410316 & 22401 \\
Déplacement max. $(\mathrm{m})$ & $2,85 \times 10^{-9}$ & $2,995 \times 10^{-9}$ & $1,33 \times 10^{-9}$ \\
\hline
\end{tabular}

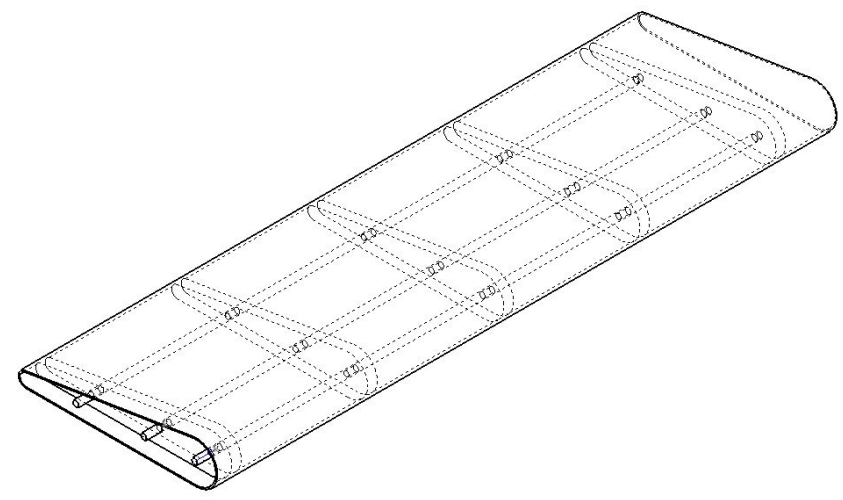

Fig. 18. Portion d'une pale d'éolienne modélisée en 3D.

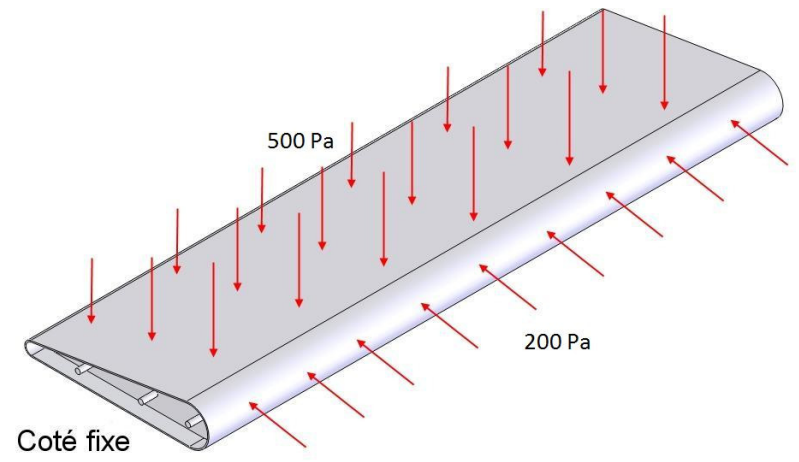

Fig. 19. Conditions appliquées sur la pale.

est un modèle entièrement modélisé à l'aide d'éléments tétraédriques linéaires et maillé avec une taille d'éléments identique à celle utilisée pour le modèle multidimensionnel.

On constate, à partir des résultats de ce tableau, que le modèle multidimensionnel se compare avantageusement avec le modèle 3D raffiné tout en présentant énormément moins de DDL. Bien qu'on puisse observer de faibles discontinuités incontournables au niveau des interfaces dimensionnelles, dues à l'utilisation de mini-poutres de rigidité très élevée, celles-ci s'avèrent négligeables et n'ont pas d'effet dès que l'on s'éloigne légèrement de ces interfaces dimensionnelles.

\subsection{Section de pale}

L'exemple suivant illustre des connexions de type poutre-coque et coque-coque sur une section de pale d'un modèle réduit (de dimensions approximatives $10 \mathrm{~mm}$ par

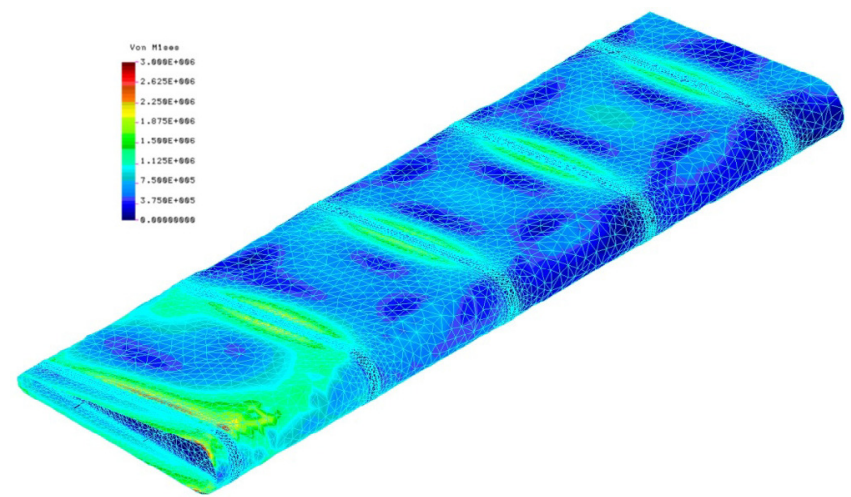

Fig. 20. Résultats de contrainte Von Mises obtenues sur la pale.

$6 \mathrm{~mm}$ par $3 \mathrm{~mm}$ ). La figure 18 illustre le modèle 3D, celui-ci comporte un revêtement en coque avec cinq renforts modélisés en coque et trois poutres de support. Le revêtement et les renforts sont en aluminium et les poutres longitudinales sont en acier.

La figure 19 illustre les conditions aux limites imposées sur la pale sous la forme de deux pressions imposées (500 $\mathrm{Pa}$ verticalement et $200 \mathrm{~Pa}$ horizontalement) et du blocage du côté gauche. La figure 20 montre les contraintes Von Mises (en $\mathrm{Pa}$ ) obtenues après les opérations de découpage, de maillage et de calcul.

\section{4 Études de variantes d'idéalisation}

Cet exemple illustre, sur un cas de structure assez simple, l'application de la méthode proposée dans le cas de plusieurs variantes d'idéalisation du modèle 3D. Le modèle 3D de la structure considérée avec les conditions aux limites imposées, ainsi que la première idéalisation considérée sont illustrés à la figure 21. Il s'agit d'une structure en acier assez simple de 5,3 mètres de largeur par 2,8 mètres de hauteur et 2,3 mètres de profondeur. Dans cet exemple, l'objectif de calcul est de déterminer plus particulièrement la contrainte dans une des quatre plaques d'ancrage au sol tel qu'identifié sur la figure 21. Pour ce faire on construit un modèle multidimensionnel où on modélise uniquement le pied (la plaque d'ancrage au sol) en 3D alors que l'on utilise des poutres pour le reste de la structure. Cette hypothèse ignore l'effet des soudures à la base de la structure ainsi que les détails des contraintes au niveau des connexions entre les poutres. Il est à noter que, sur cet exemple, la symétrie ne peut être utilisée, car les conditions aux limites ne sont pas symétriques. La répartition de contrainte de Von Mises obtenue sur ce modèle est illustrée à la figure 22 . 

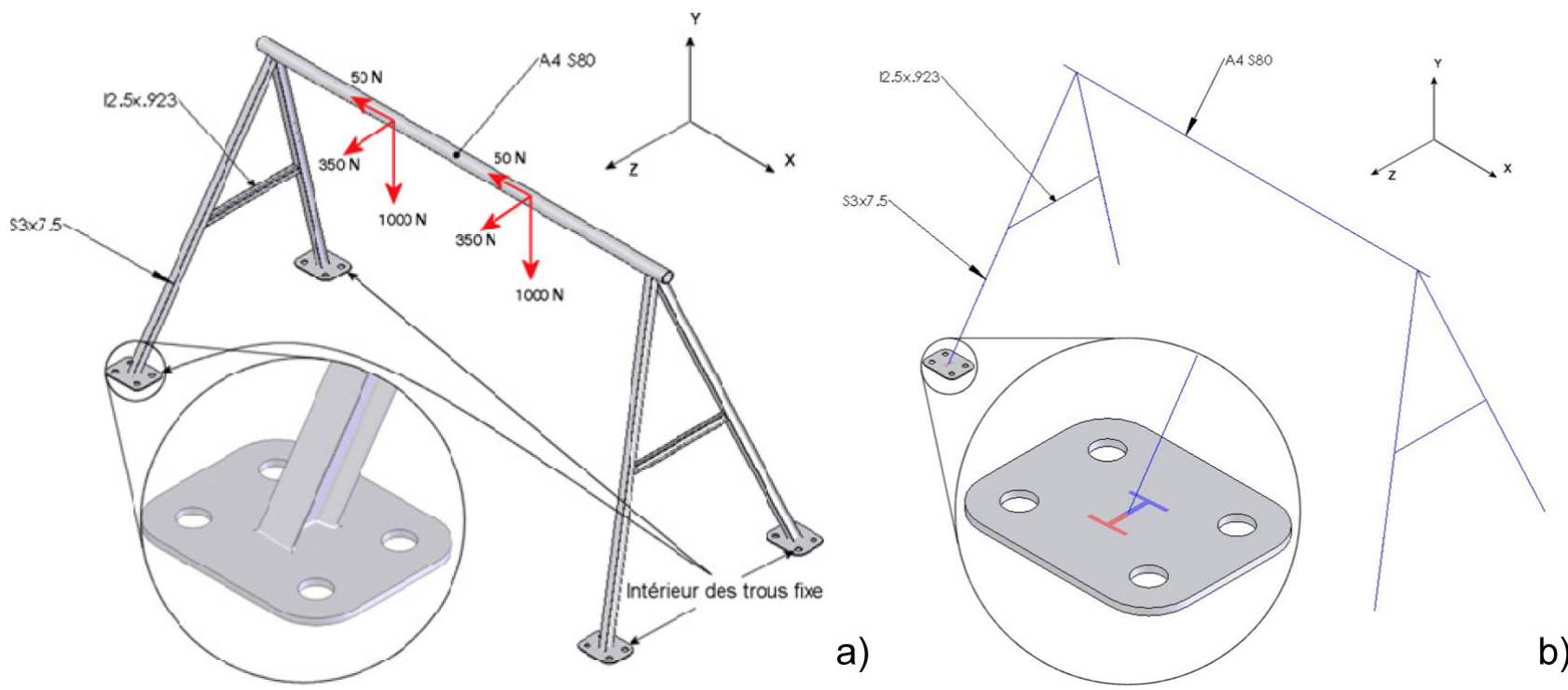

Fig. 21. (a) Structure à l'étude, (b) idéalisation.

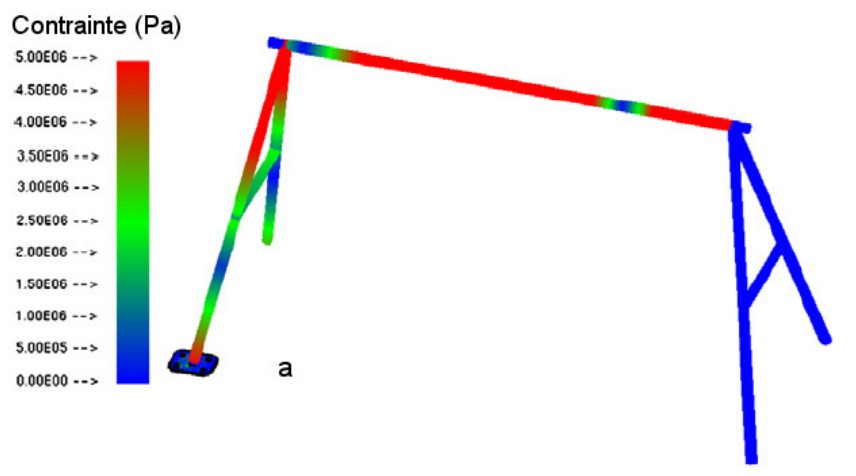

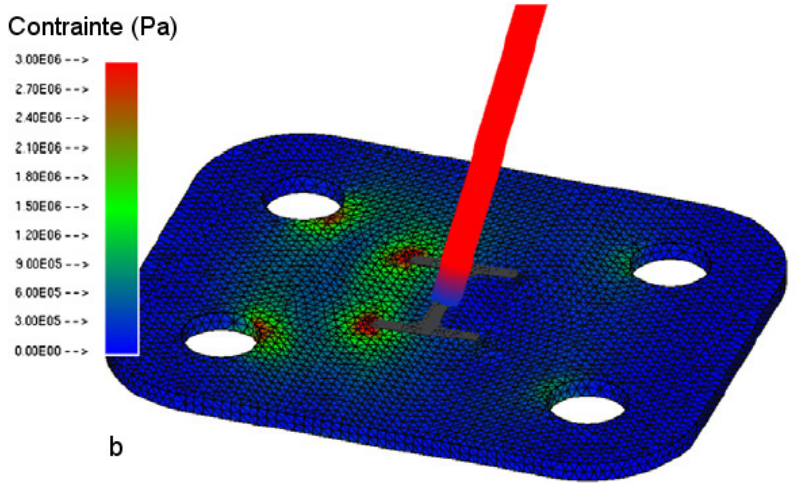

Fig. 22. Contrainte Von Mises dans le modèle, (a) structure entière, (b) pied à l'étude.

Comme il est possible de le constater sur la figure, la contrainte maximale se retrouve à la jonction entre la poutre et la plaque inférieure du pied. Si on désire calculer plus finement la contrainte à cet endroit, entre autres pour évaluer l'impact des soudures, on doit procéder à une autre idéalisation. Cette seconde idéalisation inclut une modélisation partielle de la poutre en 3D sur une courte distance. Les résultats de contrainte de Von Mises obtenus en appliquant notre méthode à cette nouvelle

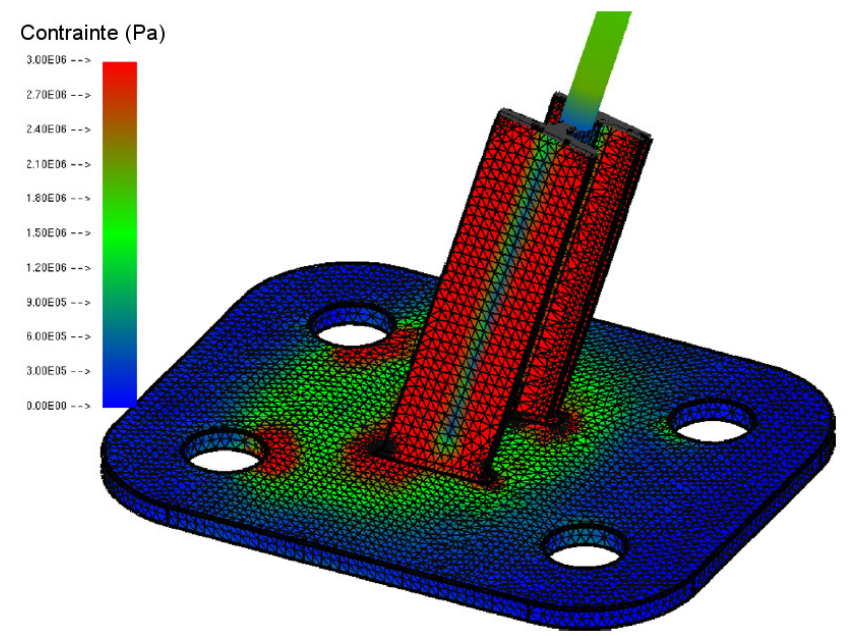

Fig. 23. Contrainte de Von Mises dans le pied à l'étude, seconde idéalisation

idéalisation sont illustrés à la figure 23. On constate alors que la contrainte est effectivement élevée dans les soudures. Ce nouveau modèle utilise une approche multidimensionnelle pour laquelle la zone critique a été modélisée en 3D afin d'évaluer précisément les contraintes dans la soudure. Cet exemple soulève la question de la pertinence de l'idéalisation selon l'objectif de calcul fixé au départ. En effet, il n'y a pas, de manière générale, de bonne ou de mauvaise idéalisation dans l'absolu. L'évaluation de la pertinence d'une idéalisation donnée doit se faire par rapport à un objectif précis du calcul par éléments-finis. La première idéalisation présentée sur cet exemple peut être tout à fait suffisante si l'objectif de calcul est d'évaluer la répartition des contraintes dans les éléments structuraux et dans la plaque d'ancrage, assez loin de l'interface dimensionnelle. Par contre, cette première idéalisation n'est pas suffisante si l'objectif de calcul est d'évaluer les 


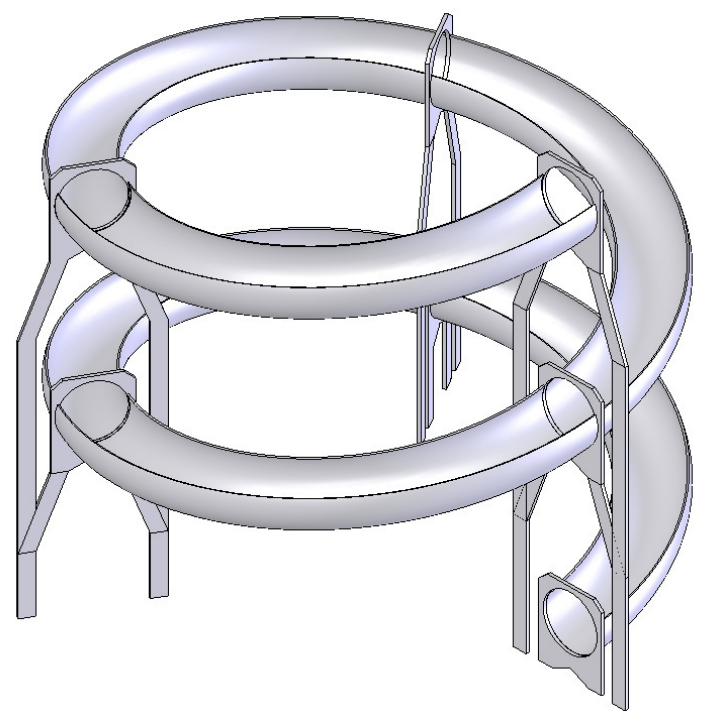

Fig. 24. Modèle solide initial.

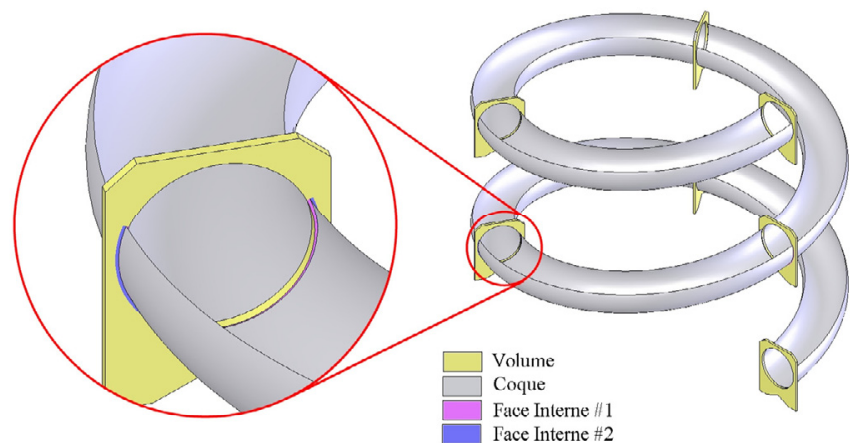

Fig. 25. Modèle idéalisé.

contraintes dans les soudures. Tout cela implique que l'automatisation de l'idéalisation de modèles CAO, en vue de la génération de modèles éléments-finis multidimensionnels, est a priori une tâche très complexe puisque l'on doit prendre en compte et donc modéliser l'objectif du calcul.

\subsection{Exemple de structure avec connexions multiples}

Ce dernier exemple illustre un modèle de glissade en aluminium. La figure 24 illustre la structure à modéliser. Pour cette étude la glissade elle-même est modélisée avec des éléments de coque alors que les supports sont modélisés avec des éléments volumiques. On lui applique une pression verticale uniforme et on bloque complètement la base de tous les supports.

La figure 25 illustre le modèle idéalisé après l'opération de découpage des faces. Sur ce modèle les conditions aux limites et les propriétés des coques (les épaisseurs) sont ensuite spécifiées. La figure 26 illustre le maillage multidimensionnel obtenu à l'étape suivante. Les 7 volumes et

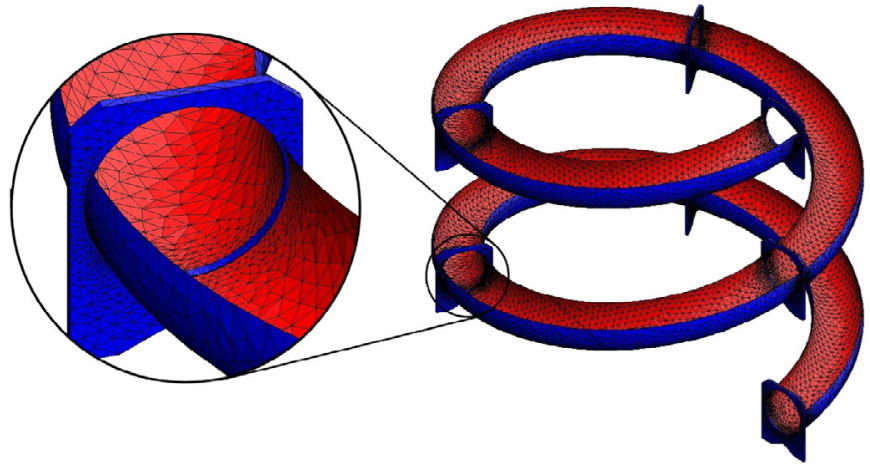

Fig. 26. Maillage du modèle idéalisé.

6 coques sont maillés indépendamment. Grâce aux traitements effectués dans la méthode, l'arête commune entre une coque et un volume n'est maillée qu'une seule fois et les nœuds ainsi générés appartiennent donc aux 2 types d'éléments à la fois.

Comme décrit précédemment, des mini-poutres orientées sont ensuite superposées à chaque segment des triangles composant le maillage des interfaces dimensionnelles.

On peut finalement procéder à l'étape du calcul. On transfère automatiquement sur le maillage obtenu à l'étape précédente toute l'information concernant les conditions aux limites et les charges, les propriétés d'épaisseur des éléments de coques et les propriétés de section et d'orientation des mini-poutres. Toute cette information (maillage, charges, conditions aux limites et propriétés) peut ensuite être exportée vers n'importe quel solveur externe. Les résultats de répartition de contrainte de Von Mises sont enfin illustrés à la figure 27.

\section{Conclusion}

Cet article présente une méthode automatique de couplage dans le cadre de l'analyse par EF multidimensionnelle, n'utilisant que des arrangements particuliers d'éléments-finis classiques (poutres, coques, éléments volumiques).

Soulignons d'abord que l'approche proposée présente les avantages suivants :

- la méthode s'applique, avec certaines variantes, à toutes les catégories de connexion;

- la précision des résultats au niveau de l'analyse par EF est nettement meilleure qu'en utilisant d'autres approches de modélisation des interfaces dimensionnelles ;

- la méthode est complètement automatique en partant d'un modèle idéalisé;

- la méthode étant essentiellement basée sur des traitements géométriques peut a priori utiliser n'importe quel mailleur automatique;

- la méthode n'utilisant que des éléments-finis classiques, le modèle EF obtenu peut être transféré facilement à n'importe quel solveur EF ; 


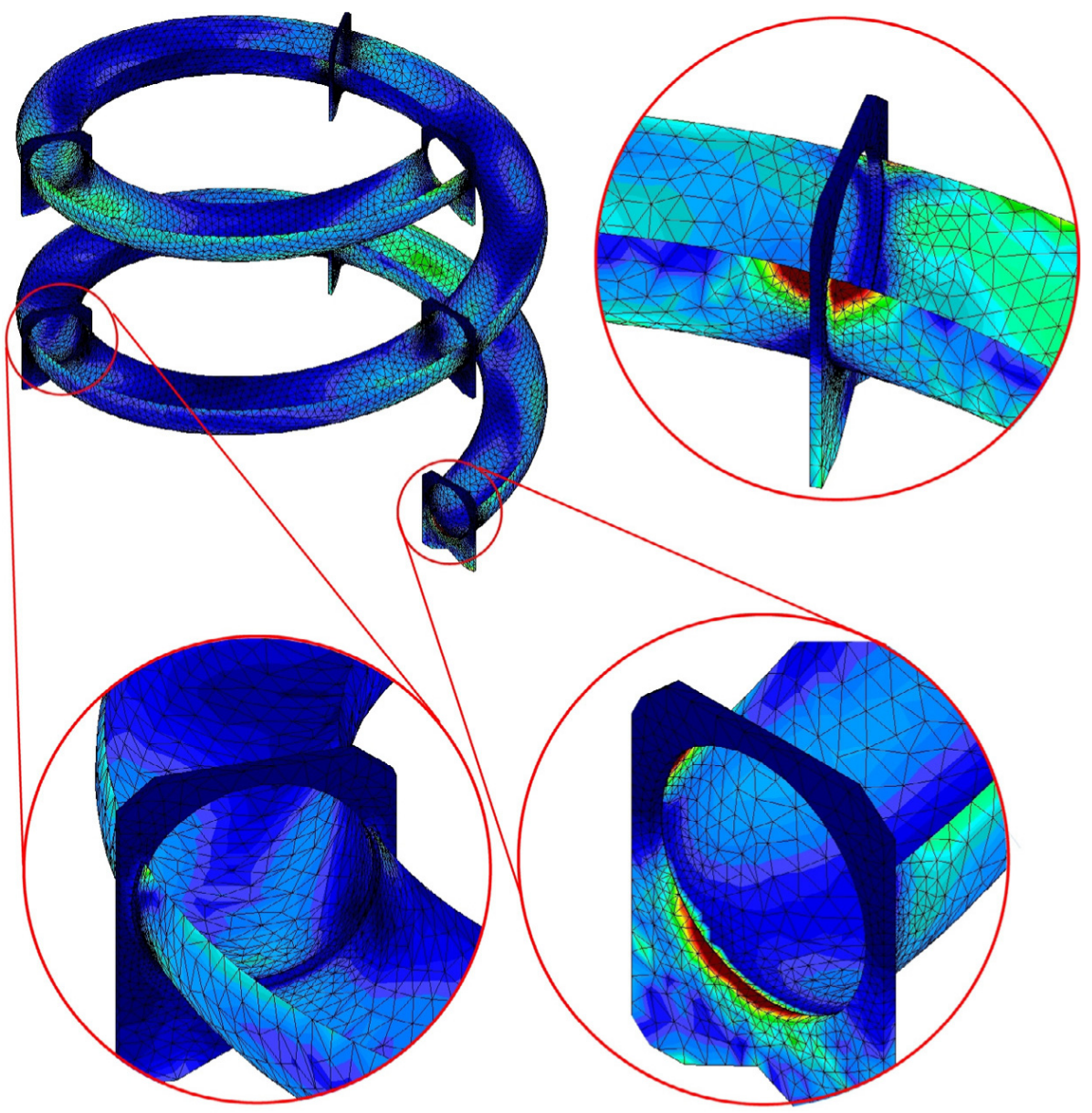

Fig. 27. Contrainte Von Mises (pour les éléments de coque, on illustre les contraintes sur la face externe de la coque).

- cette méthode ne requiert donc ni l'utilisation d'équations de contraintes, ni l'utilisation d'élémentsfinis spécifiques;

- la méthode permet la modélisation explicite de la géométrie des interfaces dimensionnelles qui permet d'appréhender plus finement les contraintes dans ces zones.

L'implémentation informatique des concepts proposés a montré leur consistance et que le processus peut être complètement automatisé en partant d'un modèle idéalisé, enrichi des données techniques pertinentes (épaisseurs des parois, propriétés et orientation des sections, conditions aux limites et carte de taille d'éléments).

Dans une boucle d'optimisation de conception, cette méthode, essentiellement basée sur des traitements géométriques, permet d'obtenir rapidement des résultats d'analyse de précision très satisfaisante et en plus, dans de nombreux cas de figure, l'analyse multidimensionnelle permet d'obtenir des résultats plus précis qu'un modèle n'utilisant que des éléments volumiques. Ceci est particulièrement vrai dans le cas de composants présentant de petites sections et de faibles épaisseurs. Généralement, l'utilisation de modèles uniquement volumiques pour des structures composées majoritairement de poutres et coques produit des maillages $3 \mathrm{D}$, soit très pauvres (en terme de qualité d'éléments et donc de résultats d'analyse), soit excessivement lourds (en terme de DDL), en comparaison de ceux obtenus à partir modèles multidimensionnels.

Il reste à raffiner la modélisation des interfaces dimensionnelles au niveau de la rigidité. En effet, l'approche proposée permet bien de modéliser précisément la forme des interfaces dimensionnelles et d'obtenir des résultats en contrainte beaucoup plus précis dans ces zones qu'en utilisant des approches conventionnelles. Par contre nous estimons qu'une modélisation encore plus fine de ces interfaces est possible en modélisant plus précisément la rigidité mécanique de l'interface que ce n'est le cas dans l'état actuel de la recherche. D'autre part, nous constatons qu'une corrélation existe entre les propriétés des mini-poutres, l'énergie de déformation emmagasinée dans les mini-poutres et la précision des résultats obtenus. Ceci permet de penser que l'analyse fine de cette énergie pourrait conduire à un ajustement plus optimal des propriétés des mini-poutres. Ceci dit, l'ajustement des propriétés des mini-poutres reste un problème assez complexe car, pour une interface dimensionnelle donnée, cet ajustement dépend nécessairement de la manière dont est sollicitée l'interface. L'utilisation conjointe de mini-poutres et 
de mini-coques, au niveau des interfaces dimensionnelles, constitue peut-être une piste de solution complémentaire.

Bien entendu, l'usage efficace de l'analyse multidimensionnelle est aussi directement relié à la capacité de produire, rapidement et facilement, des modèles idéalisés à partir de modèles solides paramétriques par caractéristiques. Un travail de recherche important est actuellement en cours, visant à mettre en place des outils, rapides et efficaces, de préparation de modèles CAO pour l'analyse par éléments-finis et l'idéalisation automatique de modèles $\mathrm{CAO}$ est un des défis majeurs à relever dans ce contexte. Comme mentionné précédemment, cette tâche est très complexe et son automatisation très ambitieuse, compte tenu du fait que, pour un exemple donné, la pertinence d'une approche d'idéalisation doive être évaluée en fonction d'un objectif de calcul précis et non simplement en fonction de critères purement géométriques.

Remerciements. Cette étude a été menée dans le cadre d'un programme de recherche subventionné par le Fonds Québécois de la Recherche sur la Nature et les Technologies (FQRNT) et par le Conseil de Recherches en Sciences Naturelles et en Génie du Canada (CRSNG).

\section{Références}

[1] C.G. Armstrong, S. Bridgett, R. Donaghy, W. Mc Cune, R. McKeag, D. Robinson, Techniques for interactive and automatic idealisation of cad models, numerical grid generation in computational field simulations, Greenwich, UK, 1998, pp. 643-662

[2] C.S. Chong, A.S. Kumar, K.H. Lee, Automatic solid decomposition and reduction for non-manifold geometric model generation, Comput. Aided Design 36 (2004) 13571369

[3] R.J. Donaghy, W. McCune, S.J. Bridgett, C.G. Armstrong, D.J. Robinson, R.M. McKeag, Dimensional Reduction of Analysis Models, in: S.N. Laboratories (éd.), International Meshing Roundtable, Pittsburgh, Pennsylvania, 1996, pp. 307-320

[4] D.J. Monaghan, I.W. Doherty, D. McCourt, C.G. Armstrong, Coupling 1D Beams to 3D Bodies, International Meshing Roundtable, Dearborn, USA, 1998, pp. 1-8
[5] K. Suresh, Generalization of the Kantorovich Method of Dimensional Reduction, International Meshing Roundtable, Santa Fe, New Mexico, 2003, pp. 261-270

[6] M. Mantyla, An introduction to solid modeling, 1988

[7] M.E. Mortenson, Geometric Modeling, 1985

[8] W. Mc Cune, C.G. Armstrong, D. Robinson, Mixeddimensional coupling in finite element models, Int. J. Numer. Methods Eng. 49 (2000) 725-750

[9] K. Shim, D.J. Monaghan, A. CG, Mixed dimensional coupling in finite element stress analysis, International Meshing Roundtable Newport Beach, California 2001, pp. 269-277

[10] H. Ben Dhia, Approches Locales-Globales Méthode Arlequin, $5^{\mathrm{e}}$ Colloque National de Calcul des Structures, Giens, France, 2005, pp. 21-32

[11] J.-C. Craveur, D. Marceau, De la CAO au calcul, Dunod, Paris, 2001

[12] D.J. Allman, A compatible triangular element including vertex rotations for plane elasticity analysis, Comput. Struct. 19 (1984) 1-8

[13] T.P. Pawlak, S.M. Yunus, Solid elements with rotational degrees of freedom: Part II - tetrahedron elements, Int. J. Numer. Methods Eng. 31 (1991) 593-610

[14] K.Y. Sze, Y.S. Pan, Hybrid stress tetrahedral elements with Allman's rotational D.O.F.s, Int. J. Numer. Methods Eng. 48 (2000) 1055-1070

[15] S. Bournival, J.-C. Cuilliere, V. Francois, A mesh-based method for coupling 1D and 3D finite elements, Design and Modelling of Mechanical Systems, Monastir, Tunisia, 2007

[16] P.J. Frey, P.-L. George, Maillages applications aux elements finis, Hermes Sciences, Paris, 1999

[17] J.-C. Cuilliere, An adaptive method for the automatic triangulation of 3D parametric surfaces, Comput. Aided Design 30 (1998) 139-149

[18] V. Francois, J.-C. Cuilliere, An a priori adaptive 3D advancing front mesh generator integrated to solid modeling, Recent Advances in Integrated Design and Manufacturing in Mechanical Engineering. (2003) 337346

[19] S. Bournival, J.-C. Cuillière, V. François, A meshgeometry based method for coupling $1 \mathrm{D}$ and 3D elements, Adv. Eng. Softw. 41 (2010) 838-858

[20] J.-C. Cuillière, S. Bournival, V. François, A meshgeometry-based solution to mixed-dimensional coupling, Comput. Aided Design 42 (2010) 509-522 